\title{
FUSION REACTIONS IN MULTICOMPONENT DENSE MATTER
}

\author{
D. G. Yakovlev \\ Ioffe Physico-Technical Institute, Poliekhnicheskaya 26, 194021 Saint-Petersburg, Russia \\ Department of Physics \& The Joint Institute for Nuclear Astrophysics, \\ University of Notre Dame, Notre Dame, IN 46556 USA \\ L. R. Gasques and M. Beard, M. Wiescher \\ Department of Physics \& The Joint Institute for Nuclear Astrophysics, \\ University of Notre Dame, Notre Dame, IN 46556 USA
}

\author{
A. V. Afanasjev \\ Department of Physics and Astronomy, Mississippi State University, P.O. Drawer 5167, MS 39762-5167 USA
}

\begin{abstract}
We analyze thermonuclear and pycnonuclear fusion reactions in dense matter containing atomic nuclei of different types. We extend a phenomenological expression for the reaction rate, proposed recently by Gasques et al. 11] for the one-component plasma of nuclei, to the multi-component plasma. The expression contains several fit parameters which we adjust to reproduce the best microscopic calculations available in the literature. Furthermore, we show that pycnonuclear burning is drastically affected by an (unknown) structure of the multi-component matter (a regular lattice, a uniform mix, etc.). We apply the results to study nuclear burning in a ${ }^{12} \mathrm{C}^{16} \mathrm{O}$ mixture. In this context we present new calculations of the astrophysical $S$-factors for carbon-oxygen and oxygenoxygen fusion reactions. We show that the presence of a CO lattice can strongly suppress carbon ignition in white dwarf cores and neutron star crusts at densities $\rho \gtrsim 3 \times 10^{9} \mathrm{~g} \mathrm{~cm}^{-3}$ and temperatures $T \lesssim 10^{8} \mathrm{~K}$.
\end{abstract}

\section{INTRODUCTION}

Nuclear reactions are most important for the physics of stars. They determine hydrogen burning in mainsequence stars, helium burning in red giants, and carbon, neon, and oxygen burning at later stages. They determine also nucleosynthesis in shock driven stellar explosions, such as type II supernovae, as well as ignition and burning in accreting stars.

Steady-state and explosive thermonuclear carbon burning during late stellar evolution [1] and in shock fronts of type II supernovae [2] is governed by the ${ }^{12} \mathrm{C}+{ }^{12} \mathrm{C}$ and possibly by the ${ }^{12} \mathrm{C}+{ }^{16} \mathrm{O}$ fusion processes. Similarly, thermonuclear oxygen burning is mainly determined by the ${ }^{16} \mathrm{O}+{ }^{16} \mathrm{O}$ and possibly by the ${ }^{16} \mathrm{O}+{ }^{20} \mathrm{Ne}$ reactions [3]. The ignition and nucleosynthesis during these burning phases critically depend on the initial fuel abundance and on the thermonuclear reaction rates.

In high-density cores of white dwarfs and crusts of neutron stars, the thermonuclear reactions are strongly affected by plasma effects (especially important for carbon ignition in cores of accreting massive white dwarfs for triggering type Ia supernova explosions). The ignition conditions are sensitive to the ${ }^{12} \mathrm{C}$ and ${ }^{16} \mathrm{O}$ abundance and to the fusion reaction rates $[4]$.

Carbon ignition has also been suggested as a trigger of superbursts in surface layers of accreting neutron stars [5]. However, the required ignition conditions seem to disagree with the observed superburst lightcurves [6]. Alternative explanations are presently being discussed, such as carbon ignition in the crust of an accreting strange (quark) star, to accommodate the observed light curve characteristics [7]. While in most of the scenarios pure carbon burning dominates the energy production, ignition conditions and associated nucleosynthesis are affected by the presence of other elements.

Pycnonuclear burning occurs in dense and cold cores of white dwarfs [8] and in crusts of accreting neutron stars 9, 10]. Theoretical formalism has been mostly developed for pycnonuclear reactions between equal nuclei, but one often needs to consider a multi-component matter, for instance, carbon-oxygen cores of white dwarfs.

In a previous publication [11] we have focused on fusion reactions between equal nuclei in a one-component plasma (OCP) of atomic nuclei (ions). In the present work we expand the study towards a multi-component plasma (MCP). The problem has two aspects; the first one is associated with the underlying nuclear physics while the second one is concerned with the plasma physics. The nuclear part deals with the reliable determination of astrophysical $S$-factors at stellar energies. These energies are low (typically, lower than a few $\mathrm{MeV}$ ), in particular if compared to the presently accessible range of low-energy fusion experiments. This prevents direct measurements of $S$-factors at laboratory conditions. Thus, one needs to calculate the $S$-factors theoretically and use these results to extrapolate measured $S$-factors towards lower stellar energy range. In Section [1] we present calculations of the $S$-factors for the two reactions of astrophysical importance, ${ }^{12} \mathrm{C}+{ }^{16} \mathrm{O}$ and ${ }^{16} \mathrm{O}+{ }^{16} \mathrm{O}$.

The plasma physics problem consists in calculating the Coulomb barrier penetration in nuclear reactions taken into account Coulomb fields of surrounding plasma particles. These fields modify the reaction rates and lead to five nuclear burning regimes [8] (two thermonuclear 
regimes, with weak and strong plasma screening; two pycnonuclear regimes, for zero-temperature and thermally enhanced burning; and the intermediate regime). These regimes are described in Section III their validity conditions are specified in Section IIIA In ordinary stars, nuclear burning proceeds in the weak screening thermonuclear regime [12, 13]. The foundation of the theory of thermonuclear burning with strong plasma screening was laid by Salpeter 14]. The strict theory of pycnonuclear burning was developed by Salpeter and Van Horn 8]. References to other works can be found in Section III and in [1].

In Section Џwe analyze calculations of Coulomb barrier penetration in MCP and propose a phenomenological expression for a reaction rate valid in all five regimes for any non-resonant fusion reaction. This expression accurately reproduces well known results in thermonuclear regimes and gives a reasonable description of the Coulomb tunneling problem in other regimes. It is important for incorporating the plasma physics effects into computer codes which simulate nucleosynthesis, especially at high densities in compact stars, such as white dwarfs and neutron stars (see above). In Section IV we illustrate the results of Sections [I] and [II by analyzing nuclear burning in ${ }^{12} \mathrm{C}^{16} \mathrm{O}$ mixtures, with the emphasis on the carbon ignition curve.

\section{ASTROPHYSICAL $S$-FACTORS FOR CARBON-OXYGEN MIXTURES}

In order to study nuclear burning in dense stellar carbon-oxygen matter (Section IV] we need fusion cross sections (or associated astrophysical factors) for three reactions, ${ }^{12} \mathrm{C}+{ }^{12} \mathrm{C},{ }^{12} \mathrm{C}+{ }^{16} \mathrm{O}$, and ${ }^{16} \mathrm{O}+{ }^{16} \mathrm{O}$. For calculating the cross sections we employ the one-dimensional barrier penetration (BP) formalism [15] and adopt the São Paulo potential [16, 17, 18, 19] to describe the real part of the nuclear interaction $V_{N}(r, E)$ :

$$
V_{N}(r, E)=V_{S P}(r, E)=V_{F}(r) \mathrm{e}^{-4 \mathrm{v}^{2} / c^{2}} .
$$

Here, $V_{F}(r)$ is the density-dependent double-folding potential, $c$ is the speed of light, $E$ is the particle collision energy (in the center-of-mass reference frame), $\mathrm{v}$ is the local relative velocity of two nuclei 1 and 2 ,

$$
\mathrm{v}^{2}(r, E)=(2 / \mu)\left[E-V_{C}(r)-V_{N}(r, E)\right],
$$

$V_{C}(r)$ is the Coulomb potential, and $\mu$ is the reduced mass.

In this paper, we adopt the two-parameter Fermi $(2 \mathrm{pF})$ distribution to describe the nuclear densities. The radii of these distributions are well approximated by the formula $R_{0}=1.31 A^{1 / 3}-0.84 \mathrm{fm}[19]$. The ${ }^{12} \mathrm{C}$ and ${ }^{16} \mathrm{O}$ diffuseness was taken to be $0.56 \mathrm{fm}$ and $0.58 \mathrm{fm}$, respectively. These values were extracted from heavy-ion elastic scattering data at sub-barrier and intermediate energies, by applying an unfolding method involving the São Paulo potential (see Refs. 20, 21, 22] for details).

Usually, fusion cross sections $\sigma(E)$ at low energies, typical for astrophysical conditions, are expressed in terms of the astrophysical $S$-factor

$$
S(E)=\sigma(E) E \mathrm{e}^{2 \pi \eta},
$$

where $\eta=\left(Z_{1} Z_{2} e^{2} / \hbar\right) \sqrt{\mu /(2 E)}$ is the familiar Gamow parameter; $Z_{1}$ and $Z_{2}$ are charge numbers of the nuclei. This parameterization removes from the fusion cross section the strong non-nuclear energy dependence [13, 23] associated with Coulomb barrier penetration. If $S(E)$ is a slowly varying function of $E$, it can be extrapolated to lower energies relevant to stellar burning.

The $S$-factors for all three reactions versus $E$ are shown in upper panels of Fig. 11. Solid lines are theoretical calculations using the BP model, while symbols are experimental data. The results for the $\mathrm{C}+\mathrm{C}$ reaction have already been discussed in Ref. 11] and are presented here for completeness of consideration. The data for this reaction are taken from Patterson et al. 24], Mazarakis and Stephens 25], High and Cujec [26], Rosales et al. [27], Kettner et al. [28], and Becker et al. [29]. The data for the $\mathrm{C}+\mathrm{O}$ reaction are taken from Christensen et al. [30] and Cujec and Barnes [31]. Finally, the data for the $\mathrm{O}+\mathrm{O}$ reaction are from Hulke et al. 32|, Kuronen et al. 33], Wu and Barnes 34, Kovar et al. 35, and Thomas et al. [36]. Lower panels in Fig. [1]show the Gamow-peak energy ranges versus the temperature of stellar matter in the thermonuclear burning regime; they will be discussed in Section IIIA

The sub-Coulomb-barrier resonances exhibited in the $\mathrm{C}+\mathrm{C}$ data at $E \lesssim 6 \mathrm{MeV}$ and in the $\mathrm{C}+\mathrm{O}$ data at $E \lesssim 7.7$ $\mathrm{MeV}$ cannot be reproduced in the framework of the BP model. However, the model provides an average description of the light- and heavy-ion fusion at energies below and above the barrier. Depending on the nuclear potential it also gives a satisfactory parameter-free description of the energy dependence of the $S$-factor, which seems reasonably accurate for extrapolating the experimental data into the stellar energy range.

The data sets presented by the different groups are not in perfect agreement. For instance, the two $\mathrm{C}+\mathrm{O}$ data sets agree in average, but disagree within a factor of $\sim 2$ for lowest $E$. The overall agreement between the theory and the data is $\sim 50 \%$. However, in the low-energy region, the slope of the measured cross section reported in Ref. [30] does not follow the calculated $S$-factors. It is difficult to predict where the data will lie in the energy range $E \lesssim 4 \mathrm{MeV}$. Regarding the $\mathrm{O}+\mathrm{O}$ reaction, the discrepancies between the different experimental results at sub-barrier energies are around a factor of 3 . The overall agreement between the data and the theory is $\sim 30 \%$. At the lowest measured energy the difference between the data and the theory are at most a factor of 3 . Further experiments at lower energies would help in verifying the validity of the predicted fusion cross sections. Nevertheless, it is important to highlight that the BP model 

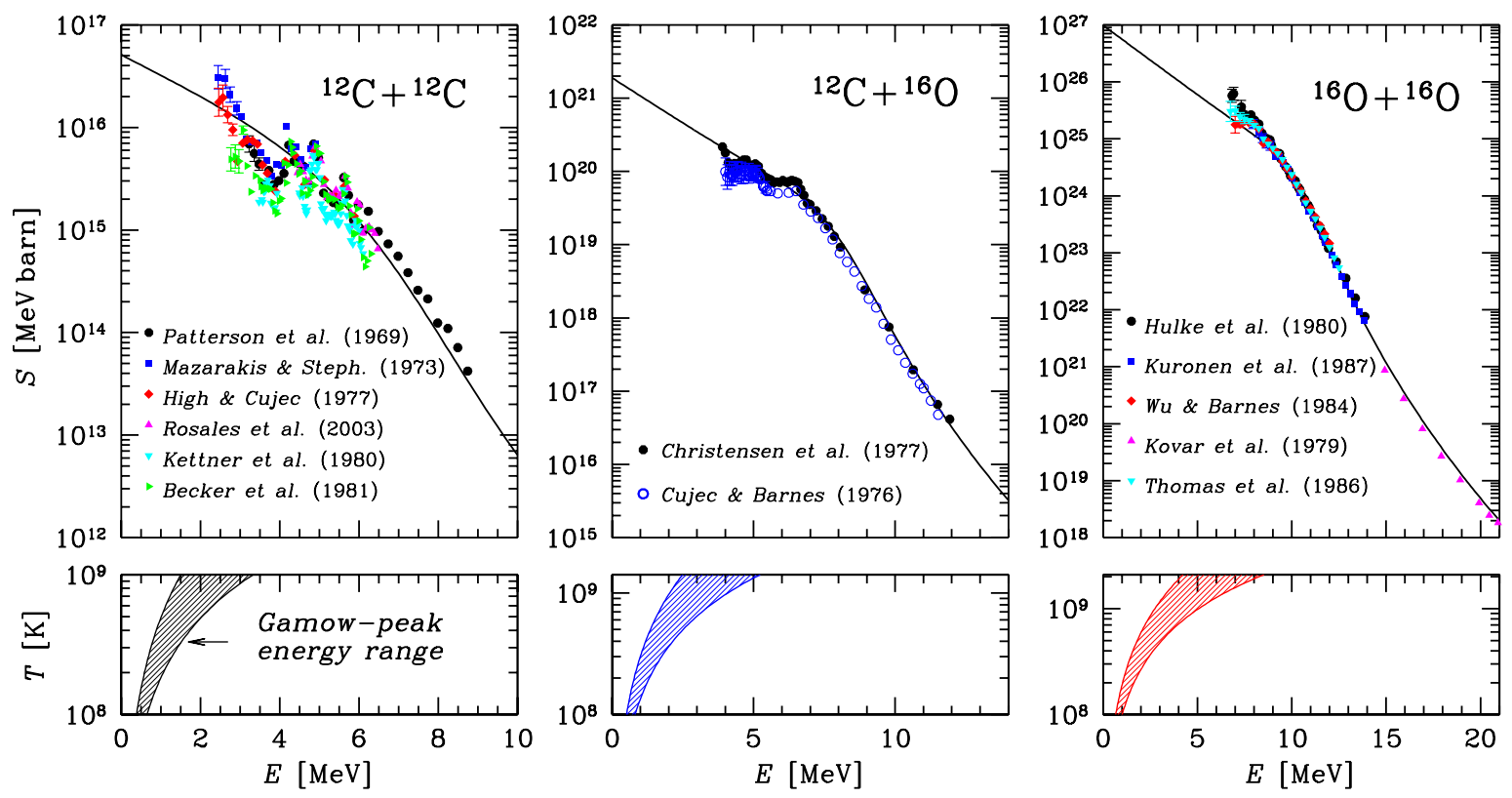

FIG. 1: (color online) Top: Astrophysical $S$-factors as a function of the center-of-mass energy $E$ for the ${ }^{12} \mathrm{C}+{ }^{12} \mathrm{C}$, ${ }^{12} \mathrm{C}+{ }^{16} \mathrm{O}$, and ${ }^{16} \mathrm{O}+{ }^{16} \mathrm{O}$ reactions. The solid lines correspond to the BP model calculations while the various symbols are experimental data. Bottom: Gamow-peak energy ranges for these reactions in the thermonuclear regime versus the temperature $T$ of stellar matter. See text for details.

does not contain any free parameter. In this sense, the $S$-factor calculations do not represent a fit to the experimental data and can be considered as a useful tool to predict average non-resonant low-energy cross sections for a wide range of fusion reactions. For many astrophysical reactions, such a description gives a reasonable estimate because the formalism of stellar reaction rates relies on the $S$-factor averaged over an entire Gamow-peak range.

The values of $S(E)$ calculated up to $E \leq 20 \mathrm{MeV}$ can be fitted by an analytic expression

$$
\begin{aligned}
S(E) & =\exp \left(A_{1}+A_{2} \Delta E\right. \\
& \left.+\frac{A_{3}+A_{4} \Delta E+A_{5} \Delta E^{2}}{1+\mathrm{e}^{-\Delta E}}\right) \mathrm{MeV} \text { barn }
\end{aligned}
$$

where $\Delta E=E-E_{0}$; the center-of-mass energy $E$ and the fit parameter $E_{0}$ are expressed in megaelectron-volts. Table【gives the fit parameters $A_{1}, \ldots, A_{5}$ and $E_{0}$ for the $\mathrm{C}+\mathrm{C}, \mathrm{C}+\mathrm{O}$ and $\mathrm{O}+\mathrm{O}$ reactions. The maximum formal fit errors are $7.2 \%$ at $E=19.8 \mathrm{Mev}$ for $\mathrm{C}+\mathrm{C} ; 6.3 \%$ at $E=7.5 \mathrm{MeV}$ for $\mathrm{C}+\mathrm{O}$; and $3.9 \%$ at $E=8.2 \mathrm{MeV}$ for the $\mathrm{O}+\mathrm{O}$ reaction. The $S$-factor for the $\mathrm{C}+\mathrm{C}$ reaction was fitted in a previous paper [11] by a slightly different expression with approximately the same accuracy. We have fitted the same data by the new expression (4) for completeness of consideration. The two fits are nearly equivalent.
TABLE I: The coefficients $A_{1}, \ldots, A_{5}$ and $E_{0}$ in the fits expression (4) for the $S$-factors of the $\mathrm{C}+\mathrm{C}, \mathrm{C}+\mathrm{O}$ and $\mathrm{O}+\mathrm{O}$ reactions.

\begin{tabular}{ccccccc}
\hline \hline Reaction & $E_{0}$ & $A_{1}$ & $A_{2}$ & $A_{3}$ & $A_{4}$ & $A_{5}$ \\
\hline${ }^{12} \mathrm{C}+{ }^{12} \mathrm{C}$ & 6.946 & 34.75 & -0.552 & -2.131 & -0.625 & 0.0315 \\
${ }^{12} \mathrm{C}+{ }^{16} \mathrm{O}$ & 8.290 & 44.32 & -0.561 & -1.480 & -0.910 & 0.0387 \\
${ }^{16} \mathrm{O}+{ }^{16} \mathrm{O}$ & 10.52 & 56.16 & -0.571 & -1.160 & -1.044 & 0.0366 \\
\hline \hline
\end{tabular}

\section{NUCLEAR FUSION RATE}

\section{A. Physical conditions and reaction regimes}

Let us consider a stellar matter which consists of ions and electrons. We assume that the ions are fully ionized and the electrons form a uniform electron background. We study a multi-component mixture of ion species $j=1,2, \ldots$, with atomic numbers $A_{j}$ and charge numbers $Z_{j}$. Let $n_{j}$ be the number density of ions $j$. The total number density of ions is $n=\sum_{j} n_{j}$; the electron number density is $n_{e}=\sum_{j} Z_{j} n_{j}$. For an OCP of ions the index $j$ will be omitted. The number density $n_{j}$ can be expressed through the mass density $\rho$ of the matter as $n_{j}=X_{j} \rho /\left(A_{j} m_{\mathrm{u}}\right)$, where $X_{j}$ is the mass fraction of ions $j$, and $m_{\mathrm{u}}=1.66055 \times 10^{-24} \mathrm{~g}$ is the atomic mass unit. In 


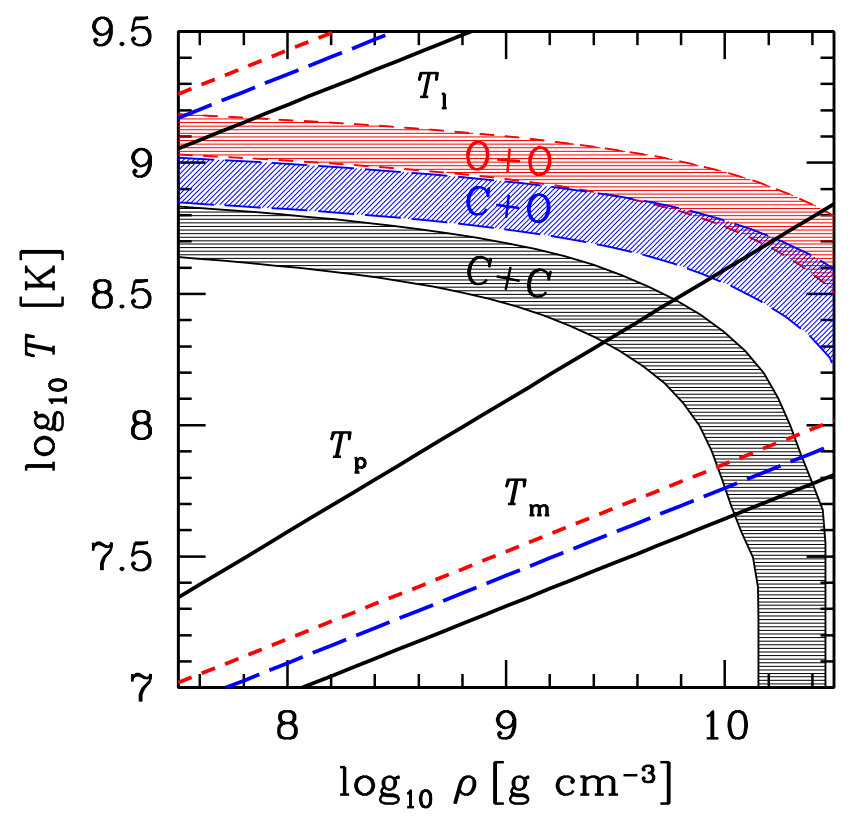

FIG. 2: (color online) Temperature-density diagram for a ${ }^{12} \mathrm{C}^{16} \mathrm{O}$ matter. Straight lines show the temperature $T_{l}$ of the appearance of ion liquid, the melting temperature $T_{m}$ of ion crystal, and the ion plasma temperature $T_{p}$. Solid lines refer to pure carbon matter, long dashes to $\mathrm{CO}$ matter with equal particle fractions of $\mathrm{C}$ and $\mathrm{O}$, and short dashes to pure oxygen matter. Three shaded strips show the regions important for $\mathrm{C}+\mathrm{C}$ burning (in pure carbon matter), $\mathrm{C}+\mathrm{O}$ burning (in $\mathrm{CO}$ mixture) and $\mathrm{O}+\mathrm{O}$ burning (in pure oxygen matter). Each strip is restricted by upper and lower lines along which the burning time equals 1 year and $10^{6}$ years, respectively (see the text for details).

a not too dense matter the total mass fraction contained in the nuclei is $X_{N}=\sum_{j} X_{j} \approx 1$. At densities higher than the neutron drip density $\left(\sim 4 \times 10^{11} \mathrm{~g} \mathrm{~cm}^{-3}\right)$, the matter contains also free neutrons; the total mass fraction contained in the nuclei is then $X_{N}<1$. It is also useful to introduce the fractional number $x_{j}=n_{j} / n$ of nuclei $j$ among other nuclei, with $\sum_{j} x_{j}=1$. Generally,

$$
\begin{aligned}
& n_{e}=n\langle Z\rangle, \quad \rho=\frac{m_{\mathrm{u}} n\langle A\rangle}{X_{N}}, \quad x_{j}=\frac{X_{j} / A_{j}}{\sum_{i} X_{i} / A_{i}}, \\
& \langle Z\rangle=\sum_{j} x_{j} Z_{j}, \quad\langle A\rangle=\sum_{j} x_{j} A_{j},
\end{aligned}
$$

where $\langle Z\rangle$ and $\langle A\rangle$ are the mean charge and mass number of ions, respectively.

Let us also introduce the Coulomb coupling parameter $\Gamma_{j}$ for ions $j$,

$$
\begin{aligned}
& \Gamma_{j}=\frac{Z_{j}^{2} e^{2}}{a_{j} k_{\mathrm{B}} T}=\frac{Z_{j}^{5 / 3} e^{2}}{a_{e} k_{\mathrm{B}} T} \\
& a_{e}=\left(\frac{3}{4 \pi n_{e}}\right)^{1 / 3}, \quad a_{j}=Z_{j}^{1 / 3} a_{e}
\end{aligned}
$$

where $T$ is the temperature, $k_{\mathrm{B}}$ is the Boltzmann constant, $a_{e}$ is the electron-sphere radius, and $a_{j}$ is the ionsphere radius (a radius of a sphere around a given ion, where the electron charge compensates the ion charge). Therefore, $\Gamma_{j}$ is the ratio of a typical electrostatic energy of the ion to the thermal energy. If $\Gamma_{j} \ll 1$ then the ions constitute an almost ideal Boltzmann gas, while for $\Gamma_{j} \gtrsim 1$ they are strongly coupled by Coulomb forces (constitute either Coulomb liquid or solid). The transformation from the gas to the liquid at $\Gamma_{j} \sim 1$ is smooth, without any phase transition. The solidification is realized as a weak second-order phase transition. According to highly accurate Monte Carlo calculations, a classical OCP of ions solidifies at $\Gamma \approx 175$ (see, e.g., Ref. [39]).

It is useful to introduce the mean ion coupling parameter $\langle\Gamma\rangle=\sum_{j} x_{j} \Gamma_{j}$. The plasma can be treated as strongly coupled if $\langle\Gamma\rangle \gtrsim 1$. This happens at $T \lesssim T_{l}$, where

$$
k_{\mathrm{B}} T_{l}=\sum_{j}\left(Z_{j}^{2} e^{2} / a_{j}\right) x_{j}=k_{\mathrm{B}} T\langle\Gamma\rangle .
$$

For low temperatures $T \ll T_{p} \ll T_{l}$ ion motion cannot be any longer considered as classical but should be quantized. Here, $T_{p}$ is the Debye (plasma) temperature associated with the ion plasma frequency $\omega_{p}$ (a typical frequency of ion vibrations in Coulomb crystals - see, e.g., Ref. [8]),

$$
T_{p}=\frac{\hbar \omega_{p}}{k_{\mathrm{B}}}, \quad \omega_{p}^{2}=\sum_{j} \frac{4 \pi Z_{j}^{2} e^{2} n_{j}}{A_{j} m_{\mathrm{u}}} .
$$

The most difficult problem of a strongly coupled MCP at low temperatures consists in understanding its actual state. Extensive Monte Carlo simulations [37] of the freezing of a classical OCP indicate that it can freeze into imperfect body-centered cubic (bcc) or faced-centered cubic (fcc) microcrystal (or microcrystals). Unfortunately, publications on reliable simulations of freezing of an MCP are almost absent. Evidently, the cold MCP is much more rich in physics than the OCP. It can be an MCP regular lattice or microcrystals (with defects); or an amorphous, uniformly mixed structure; or a lattice of one phase with random admixture of other ions. One cannot exclude an ensemble of phase separated domains. For the sake of completeness, we will consider different possibilities. An MCP obeys the linear mixing rule with the high accuracy. Accordingly, the difference in energies of the indicated states is very small and is a subject of vigorous debates (e.g., [38] and references therein). It is possible that a low-temperature MCP can be in different states depending on the history of its formation in a star with decreasing temperature.

To make our consideration less abstract, we will apply it to a ${ }^{12} \mathrm{C}^{16} \mathrm{O}$ mixture. The appropriate temperaturedensity diagram is shown in Fig. 2 We present the temperatures $T_{l}, T_{m}$ and $T_{p}$ for a pure carbon matter $\left(x_{\mathrm{C}}=1\right.$, solid lines $)$, for a mixture of equal amounts of $\mathrm{C}$ and $\mathrm{O}$ nuclei $\left(x_{\mathrm{C}}=\frac{1}{2}\right.$, long dashes) and for a pure oxygen 
matter $\left(x_{\mathrm{C}}=0\right.$, short dashes). The melting temperature of the CO mixture is taken to be $T_{m}=T_{l} / 175$. The plasma temperature $T_{p}$ is the same for all three cases. Notice that the electrons are strongly degenerate at all $\rho$ and $T$ displayed in Fig. 2 At $\rho>4 \times 10^{10} \mathrm{~g} \mathrm{~cm}^{-3}$ carbon nuclei cannot survive in dense matter because of beta captures; at $\rho>2 \times 10^{10} \mathrm{~g} \mathrm{~cm}^{-3}$ oxygen nuclei will also be destroyed by beta captures. Therefore, it is unreasonable to extend the $\mathrm{CO}$ diagram to higher densities.

We will be interested in nuclear fusion reactions

$$
\left(A_{i}, Z_{i}\right)+\left(A_{j}, Z_{j}\right) \rightarrow\left(A_{c}, Z_{c}\right),
$$

where $A_{c}=A_{i}+A_{j}$ and $Z_{c}=Z_{i}+Z_{j}$ refer to a compound nucleus $c$. For our example in Fig. 2 we have three reactions, $\mathrm{C}+\mathrm{C}, \mathrm{C}+\mathrm{O}$, and $\mathrm{O}+\mathrm{O}$ (Section $\amalg$ ). The experimental cross sections for these reactions show no very pronounced resonance structures and can be described in the framework of non-resonant reaction formalism [1, 23 as discussed above. Notice, that the data for the $\mathrm{C}+\mathrm{C}$ and $\mathrm{C}+\mathrm{O}$ reactions exhibit some resonant structures demonstrated in Fig. 1] In the lower panel of this figure we show the Gamow-peak energy range as a function of temperature for these reactions in the thermonuclear burning regime (Section 【IB ). We see that at high enough $T$ the Gamow-peak range covers the energy range, where the oscillations are experimentally measured. However, at these values of $T$ the Gamowpeak range is sufficiently wide and the energy integration in the reaction rate should smear out the oscillatory behavior. For lower $T$ the Gamow peak is narrower, but it shifts to low energies inaccessible to laboratory experiments. In the absence of experimental and theoretical data on the presence of oscillations at these low energies we will adopt the standard assumption that the reactions in question can be treated as non-resonant in applications to stellar burning.

The shaded strips Fig. 2 show the $T-\rho$ domains most important for these reactions. We will describe them in more detail in Section IV

To study a reaction (9) we introduce the so called ionsphere quantities

$$
\begin{aligned}
& a_{i j}=\frac{a_{i}+a_{j}}{2}, \quad \Gamma_{i j}=\frac{Z_{i} Z_{j} e^{2}}{a_{i j} k_{\mathrm{B}} T}, \quad T_{i j}^{(l)}=\frac{Z_{i} Z_{j} e^{2}}{a_{i j} k_{\mathrm{B}}}, \\
& T_{i j}^{(p)}=\frac{\hbar}{k_{\mathrm{B}}}\left(\frac{4 \pi Z_{i} Z_{j} e^{2} n_{i j}}{2 \mu_{i j}}\right)^{1 / 2},
\end{aligned}
$$

where $\mu_{i j}=m_{\mathrm{u}} A_{i} A_{j} / A_{c}$ is the reduced mass of the reacting nuclei, and $n_{i j}=3 /\left(4 \pi a_{i j}^{3}\right)$. Basing on the ionsphere model of a strongly coupled Coulomb plasma, one expects (e.g., Ref. [40]) that $a_{i j}$ characterizes an equilibrium distance between neighboring nuclei $i$ and $j, \Gamma_{i j}$ describes their Coulomb coupling, $T_{i j}^{(l)}$ is the temperature for the onset of strong coupling, and $T_{i j}^{(p)}$ is a local Debye temperature (for oscillations of ions $i$ and $j$ ). In an OCP we have $a_{i j}=a, \Gamma_{i j}=\Gamma, T_{i j}=T_{p}$. We will also need

$$
r_{\mathrm{B} i j}=\hbar^{2} /\left(2 \mu_{i j} Z_{i} Z_{j} e^{2}\right),
$$

which reduces to the ion Bohr radius in the case of equal ions $j=i$. In addition, we will need the parameter

$$
\begin{aligned}
\lambda_{i j}= & r_{\mathrm{B} i j}\left(\frac{n_{i j}}{2}\right)^{1 / 3}=\frac{2 r_{\mathrm{B} i j}}{\left(Z_{i}^{1 / 3}+Z_{j}^{1 / 3}\right)}\left(\frac{\rho X_{N}\langle Z\rangle}{2\langle A\rangle m_{\mathrm{u}}}\right)^{1 / 3} \\
= & \frac{A_{i}+A_{j}}{A_{i} A_{j} Z_{i} Z_{j}\left(Z_{i}^{1 / 3}+Z_{j}^{1 / 3}\right)} \\
& \times\left(\frac{\rho X_{N}\langle Z\rangle}{\langle A\rangle 1.3574 \times 10^{11} \mathrm{~g} \mathrm{~cm}^{-3}}\right)^{1 / 3}
\end{aligned}
$$

which corresponds to the parameter $\lambda$ introduced by Salpeter and Van Horn [8] in the OCP case.

In the following sections we will discuss nuclear burning in MCP for the five burning regimes introduced in Ref. [8] and analyzed in detail for OCP in our previous work [11]. We will demonstrate that the formalism developed for OCP can be adapted to more complex MCP scenarios.

\section{B. Classical thermonuclear reaction rate}

In the classical thermonuclear (weak screening) regime $\left(T \gg T_{i j}^{(l)}\right)$ the reacting ions constitute an almost ideal gas [12]. The rate for non-resonant fusion processes (such as considered here) is well known,

$$
R_{i j}^{\mathrm{th}}=\frac{4 n_{i} n_{j}}{1+\delta_{i j}} \sqrt{\frac{2 E_{i j}^{\mathrm{pk}}}{3 \mu_{i j}}} \frac{S\left(E_{i j}^{\mathrm{pk}}\right)}{k_{\mathrm{B}} T} \exp \left(-\tau_{i j}\right),
$$

where $S(E)$ is the astrophysical factor; $\delta_{i j}$ is the Kronecker delta, which excludes double counting of the same collisions in reactions with identical nuclei $(i=j)$; $E_{i j}^{\mathrm{pk}}=T k_{\mathrm{B}} \tau_{i j} / 3$ is the Gamow peak energy (the relative energy of colliding nuclei which gives the major contribution into the reaction rate) and

$$
\tau_{i j}=\left(\frac{27 \pi^{2} \mu_{i j} Z_{i}^{2} Z_{j}^{2} e^{4}}{2 k_{\mathrm{B}} T \hbar^{2}}\right)^{1 / 3}
$$

is the parameter which characterizes the Coulomb barrier penetrability. This parameter can be written as

$$
\tau_{i j}=3(\pi / 2)^{2 / 3}\left(E_{i j}^{\mathrm{a}} / k_{\mathrm{B}} T\right)^{1 / 3}, \quad E_{i j}^{\mathrm{a}} \equiv 2 \mu_{i j} Z_{i}^{2} Z_{j}^{2} e^{4} / \hbar^{2} .
$$

Then

$$
R_{i j}^{\mathrm{th}}=\frac{n_{i} n_{j}}{1+\delta_{i j}} S\left(E_{i j}^{\mathrm{pk}}\right) \frac{r_{\mathrm{B} i j}}{\hbar} P_{\mathrm{th}} F_{\mathrm{th}}
$$

where $r_{\mathrm{B} i j}$ is a convenient dimensional factor defined by Eq. (11), $F_{\text {th }}$ is the exponential function, and $P_{\text {th }}$ is the pre-exponent,

$$
F_{\mathrm{th}}=\exp \left(-\tau_{i j}\right), \quad P_{\mathrm{th}}=\frac{8 \pi^{1 / 3}}{\sqrt{3} 2^{1 / 3}}\left(\frac{E_{i j}^{\mathrm{a}}}{k_{\mathrm{B}} T}\right)^{2 / 3} .
$$


Typically, the main contribution into the reaction rate comes from suprathermal particles $\left(E_{i j}^{\mathrm{pk}} \gg k_{\mathrm{B}} T\right)$, and the Coulomb barrier is very thick $\left(\tau_{i j} \gg 1\right)$. The reaction rate decreases exponentially with decreasing $T$. Typical Gamow-peak energy ranges for the $\mathrm{C}+\mathrm{C}, \mathrm{C}+\mathrm{O}$, and $\mathrm{O}+\mathrm{O}$ reactions in the thermonuclear regimes are shown in Fig. 11 These energies are defined as $E_{i j}^{\mathrm{pk}}-\Delta E \lesssim E \lesssim$ $E_{i j}^{\mathrm{pk}}+\Delta E$, with $\Delta E \sim 2 \sqrt{E_{i j}^{\mathrm{pk}} k_{\mathrm{B}} T}$.

\section{Thermonuclear regime with strong screening}

The thermonuclear regime with strong plasma screening operates in the temperature range $T_{i j}^{(p)} \lesssim T \lesssim T_{i j}^{(l)}$, where the plasma ions constitute a strongly coupled Coulomb system (liquid or solid). The majority of ions are confined in deep Coulomb potential wells but the main contribution into the reaction rate comes from a very small amount of highly energetic suprathermal ions which are nearly free (see, e.g., Refs. 8, 14]). However, neighboring plasma ions strongly screen the Coulomb interaction between the reacting ions. The screening simplifies close approaches of the reacting ions, required for a Coulomb tunneling, and enhances thus the reaction rate (with respect to the classical thermonuclear reaction rate).

In analogy with the OCP case (e.g., Ref. [1]]), the enhancement can be included into the exponential function $F_{\text {th }}$,

$$
F_{\text {th }}=F_{\text {sc }} \exp \left(-\tau_{i j}\right), \quad F_{\text {sc }}=\exp \left(h_{i j}\right),
$$

where $F_{\mathrm{sc}}$ is the enhancement factor and $h_{i j}$ is a function of plasma parameters.

We will analyze $h_{i j}$ in the same manner as was done in Ref. 11] for the OCP. For this purpose we notice that the reacting nuclei move in the potential $W(r)=$ $Z_{i} Z_{j} e^{2} / r-H_{i j}(r)$, where $H_{i j}(r)$ is the plasma potential created by neighboring plasma ions. In the thermonuclear regime, $H_{i j}(r)$ is almost constant along a Coulomb tunneling path. Accordingly, $h_{i j}$ can be split into two terms, $h_{i j}=h_{i j}^{(0)}+h_{i j}^{(1)}$. The main term $h_{i j}^{(0)}$ is obtained assuming that $H_{i j}(r) \approx H_{i j}(0)$ is constant along a tunneling path; a small correction $h_{i j}^{(1)}$ is produced by a weak variation of the plasma potential along this path. We will discuss $h_{i j}^{(0)}$ explicitly in this section and introduce $h_{i j}^{(1)}$ phenomenologically in Section $\llbracket$ IIG when we propose an analytic approximation for the reaction rate in all regimes.

It is well known (e.g., Refs. 14, 41]) that $h_{i j}^{(0)}=$ $H_{i j}(0) / k_{\mathrm{B}} T$, where $H_{i j}(0)$ is the properly averaged (mean-field) plasma potential at $r=0$. Thus defined, $H_{i j}(0)$ becomes a thermodynamic quantity which can be expressed through the difference of the classical Coulomb free energies for a given system and a system with the two reacting nuclei merging into a compound nucleus (e.g., Ref. 41]). However, a strongly coupled classical multi-component Coulomb liquid obeys very accurately the linear mixing rule (see Ref. 38] for recent results). Using this rule, one obtains

$$
h_{i j}^{(0)}=f_{0}\left(\Gamma_{i}\right)+f_{0}\left(\Gamma_{j}\right)-f_{0}\left(\Gamma_{c}\right),
$$

where $f_{0}(\Gamma)$ is the Coulomb free energy per ion in an OCP (in units of $k_{\mathrm{B}} T$ ). This formula was derived by Jancovici 42] for an OCP and generalized by Mochkovitch for an MCP (see Ref. [43]). The function $f_{0}(\Gamma)$ is very accurately determined by Monte Carlo simulations. For instance, according to DeWitt and Slattery [44], the function $f_{0}(\Gamma)$ for a classical one-component Coulomb liquid at $1 \leq \Gamma \leq 170$ can be approximated as

$$
\begin{aligned}
f_{0}(\Gamma)= & -0.899172 \Gamma+(1 / s) 0.602249 \Gamma^{s} \\
& -0.274823 \ln \Gamma-1.401915,
\end{aligned}
$$

where $s=0.3230064$. It gives a highly accurate expression for $h_{i j}^{(0)}$, but it is inconvenient for an analytic interpolation of the reaction rate (Section II G). Instead, we will use a simpler linear expression $f_{0}(\Gamma)=-0.9 \Gamma$ provided by the ion-sphere model [14],

$$
\begin{aligned}
& h_{i j}^{(0)}=C_{i j}^{\mathrm{sc}} \Gamma_{i j}, \\
& C_{i j}^{\mathrm{sc}}=0.9\left[Z_{c}^{5 / 3}-Z_{i}^{5 / 3}-Z_{j}^{5 / 3}\right] \frac{Z_{i}^{1 / 3}+Z_{j}^{1 / 3}}{2 Z_{i} Z_{j}} .
\end{aligned}
$$

This expression seems crude but, actually, it is accurate. For a nuclear reaction in an $\mathrm{OCP}\left(Z_{i}=Z_{j}=Z_{c} / 2\right)$, this equation gives $C^{\mathrm{sc}}=1.0573$, very close to the value 1.0754 inferred [11] from Eq. (20). In the range of $1 \leq$ $\Gamma_{i j} \leq 170$ the ion-sphere model (21) gives the enhancement factor $\exp \left(h_{i j}^{(0)}\right)$ which is systematically lower than the more accurate enhancement factor, given by Eqs. (19) and (20). The maximum difference of these enhancement factors for charge ratios $1 / 5 \leq Z_{i} / Z_{j} \leq 5$ reaches $\approx 15$ at the highest value of $\Gamma_{i j}=170$. This difference can be regarded as insignificant because at such $\Gamma_{i j}$ the enhancement factor itself is as huge as $\exp \left(h_{i j}^{(0)}\right) \sim 10^{74}$. For lower $\Gamma_{i j}$ the expression (21) is more accurate. For instance, for $\Gamma_{i j}=50$ and $Z_{i} / Z_{j}=5$ it underestimates the enhancement factor only by a factor of 3 . In the range of $1 \leq \Gamma_{i j} \leq 10$ for $1 / 5 \leq Z_{i} / Z_{j} \leq 5$ the underestimation does not exceed a factor of 1.5 . Notice that in the MCP the factor $C_{i j}^{\mathrm{sc}}$ depends on $Z_{i}$ and $Z_{j}$.

Although the above approach is more accurate, one usually calculates $h_{i j}^{(0)}$ by extrapolating the mean-field plasma potential $H_{i j}(r)$, obtained from classical Monte Carlo sampling, to $r \rightarrow 0$. In particular, Ogata et al. [45, 46] used this method to study the enhancement of thermonuclear burning in the liquid phases of OCP and binary ion mixtures (BIMs). The leading term in Eqs. (19) and (20) of Ref. [46], equivalent to the leading terms (19) and (21), is $h_{i j}^{(0)}=\Gamma_{i j}\left(1.148-0.00944 \ln \Gamma_{i j}-\right.$ 
$\left.0.000168\left(\ln \Gamma_{i j}\right)^{2}\right)$. This expression was employed also by Kitamura [4] for constructing the analytic approximation for the reaction rates in OCP and BIMs in all reaction regimes (although by that time a more accurate expression was obtained by Ogata [4] for the OCP using path integral Monte Carlo simulations). Comparing the expression of Ref. 46] with (19) and (21) we see that the expression of Ref. 46] systematically overestimates the plasma enhancement. In the OCP the overestimation reaches 11] a factor of $\sim 40$ for $\Gamma=170$, which is not very significant. However, the coefficients in this expression are independent of $Z_{i}$ and $Z_{j}$, in disagreement with Eq. (21). As a result, the overestimation increases with the growth of the charge ratio $Z_{i} / Z_{j}$, reaching $\sim 150$ and $\sim 2 \times 10^{4}$ at $Z_{i} / Z_{j}=2$ and $Z_{i} / Z_{j}=5$, respectively, for $\Gamma_{i j}=170$. Therefore, when the difference of charges $Z_{i}$ and $Z_{j}$ increases, the results of Refs. [46] and [4] become less accurate. The nature of inaccuracy comes from the problems of extrapolation of $H_{i j}(r)$ to $r \rightarrow 0$. It was analyzed by Rosenfeld for the OCP [49] and the MCP [50.

Equations (20) and (21) become invalid in the regime of weak screening $\left(\Gamma_{i j} \ll 1\right.$; Section【IIB), where the well known Debye-Hückel theory should be used. In particular, the screening function in the weakly coupled MCP becomes 14]

$$
h_{i j}^{(0)}=\frac{Z_{i} Z_{j} e^{2}}{k_{\mathrm{B}} T r_{\mathrm{D}}}=\left(\frac{3 \Gamma_{e}^{3}\left\langle Z^{2}\right\rangle Z_{i}^{2} Z_{j}^{2}}{\langle Z\rangle}\right)^{1 / 2},
$$

where $r_{\mathrm{D}}$ is the ion Debye screening length and $\left\langle Z^{2}\right\rangle \equiv$ $\sum_{j} Z_{j}^{2} x_{j}$. Introducing $\Gamma_{i j}$, we obtain

$$
h_{i j}^{(0)}=\zeta_{i j} \Gamma_{i j}^{3 / 2}, \quad \zeta_{i j}=\left(\frac{3\left\langle Z^{2}\right\rangle\left(Z_{i}^{1 / 3}+Z_{j}^{1 / 3}\right)^{3}}{8\langle Z\rangle Z_{i} Z_{j}}\right)^{1 / 2} .
$$

For reactions in an OCP, we have $\zeta=\sqrt{3}$. In an MCP, $\zeta_{i j}$ depends on ion charge numbers.

A simple phenomenological interpolation which reproduces the strong and weak screening limits (Eqs. (21) and (23)) and combines them in the $\Gamma_{i j}$ range from $\sim 0.1$ to $\sim 1$ is

$$
h_{i j}^{(0)}=C_{i j}^{\mathrm{sc}} \Gamma_{i j}^{3 / 2} /\left[\left(C_{i j}^{\mathrm{sc}} / \zeta_{i j}\right)^{4}+\Gamma_{i j}^{2}\right]^{1 / 4} .
$$

Because accurate calculations of the MCP free energy of ions in this range are absent, we cannot test the accuracy of our interpolation. However, the plasma screening enhancement of reaction rates at these values of $\Gamma_{i j}$ is weak and the interpolation uncertainty does not affect strongly the reaction rates.

\section{Zero-temperature pycnonuclear regime}

This regime operates at low temperatures, $T \lesssim$ $0.5 T_{i j}^{(p)} / \ln \left(T_{i j}^{(l)} / T_{i j}^{(p)}\right)$, at which thermal effects are neg- ligible and all the ions occupy ground states in their potential wells. The Coulomb barrier is penetrated owing to zero-point vibrations of ions around their equilibrium positions. Because the vibration amplitudes are generally small, neighboring pairs of ions (closest neighbors) make the major contribution into the reaction rate.

Generalizing Eq. (35) of Salpeter and Van Horn [8] to the MCP case we can present the pycnonuclear reaction rate as (see, e.g., Eq. (7) in Ref. [4])

$$
R_{i j}^{\mathrm{pyc}}=\frac{n_{i}}{1+\delta_{i j}}\left\langle\nu_{i j} p_{i j}\right\rangle_{\mathrm{av}},
$$

where $\nu_{i j}$ is the number of nearest nuclei $j$ around a nucleus $i, p_{i j}$ is the reaction rate for a fixed pair $i j$, and the brackets $\langle\ldots\rangle_{\text {av }}$ denote statistical averaging over an ensemble of such pairs. For instance, it is currently thought that the OCP of ions at zero temperature forms a bcc crystal. In this case, any ion is surrounded by 8 closest neighbors, with the equilibrium distance between them $d=\left(3 \pi^{2}\right)^{1 / 6} a$, where $a$ is the ion-sphere radius.

According to Eqs. (35), (37), and (39) of Ref. 8], the reaction rate for a pair of neighboring ions in an $\mathrm{OCP}$ can be written as

$$
p=D_{\mathrm{pyc}} \frac{\lambda^{3-C_{\mathrm{pl}}} S\left(E^{\mathrm{pk}}\right)}{\hbar r_{\mathrm{B}}^{2}} \exp \left(-\frac{C_{\mathrm{exp}}}{\sqrt{\lambda}}\right),
$$

where $\lambda$ and $r_{\mathrm{B}}$ are given by Eqs. (12) and (11), respectively (for the OCP); while $D_{\mathrm{pk}}, C_{\mathrm{pl}}$ and $C_{\text {exp }}$ are constants which depend on a Coulomb barrier penetration model and on the lattice type. Finally, the characteristic reaction energy is $E^{\mathrm{pk}} \sim \hbar \omega_{p}=k_{\mathrm{B}} T_{p}$, where $\omega_{p}$ is the ion plasma frequency defined by Eq. (8) (a typical frequency of zero-point ion vibrations).

Salpeter and Van Horn [8] used the WKB approximation and considered two models of Coulomb barrier penetration in the bcc lattice, the static and relaxed lattice ones, to account for the lattice response to a motion of tunneling nuclei. Later Schramm and Koonin [9] extended this consideration taking into account the dynamical effect of motion of surrounding ions in response to the motion of the tunneling nuclei in the relaxed lattice. In addition, they considered the fcc Coulomb lattice. The results of Refs. [8, 9] are analyzed in Ref. [11. Note that the pycnonuclear burning rates for fcc or bcc OCP crystals are very similar. We expect that for an amorphous OCP they are of the same order of magnitude.

Pycnonuclear reactions in the MCP require complicated calculations (which, hopefully, will be done in the future). We will restrict ourselves to a simpler consideration based on similarity criteria and some general assumptions. Even a state of the MCP at low temperatures is not clear. It can be a regular lattice (with defects); a uniformly mixed state; an ensemble of phase-separated domains, etc. (Section ஹA). It can also be a combination of these states. 


\section{Uniformly mixed $M C P$}

Let us start with a uniformly mixed MCP. An obvious generalization of Eq. (26) to the MCP would be to replace $r_{\mathrm{B}} \rightarrow r_{\mathrm{B} i j}$ and $\lambda \rightarrow \lambda_{i j}$ in accordance with Eqs. (11) and (12). This replacement should correctly reflect the rescaling of inter-ion distances and oscillator frequencies in an MCP within the ion-sphere model (see, e.g., Ref. [51]).

However, the rescaling may be not exact. We will take this into account by adopting a simplified assumption that any pair of close neighbors behaves as an elementary oscillator with an equilibrium separation $d_{i j}$ and an oscillator frequency $\omega_{i j}$. For an OCP we have $\lambda \sim \hbar^{2} /\left(A m_{\mathrm{u}} d^{2} \omega\right)^{2}$ and $\omega \sim \omega_{p}$. In the MCP we expect to have $\lambda \rightarrow \widetilde{\lambda}_{i j} \sim \hbar^{2} /\left(2 \mu_{i j} d_{i j}^{2} \omega_{i j}\right)^{2}$, where $d_{i j}$ and $\omega_{i j}$ are the actual equilibrium distance and the effective oscillator frequency, respectively. With this replacement from Eq. (26) we obtain

$$
p_{i j}=D_{\mathrm{pyk}} \frac{\widetilde{\lambda}_{i j}^{3-C_{\mathrm{pl}}} S\left(E_{i j}^{\mathrm{pk}}\right)}{\hbar r_{\mathrm{B} i j}^{2}} \exp \left(-\frac{C_{\mathrm{exp}}}{\left(\widetilde{\lambda}_{i j}\right)^{1 / 2}}\right) .
$$

Now we assume that the actual values $d_{i j}$ and $\omega_{i j}$ can deviate from the ion-sphere values $d_{i j}^{(0)}$ and $\omega_{i j}^{(0)}$, and introduce the quantities

$$
\alpha_{\mathrm{d} i j}=d_{i j} / d_{i j}^{(0)}, \quad \alpha_{\omega i j}=\omega_{i j} / \omega_{i j}^{(0)},
$$

which measure the deviations. They will be treated as parameters to be varied within reasonable limits. Then

$$
\widetilde{\lambda}_{i j}=\lambda_{i j} \alpha_{\lambda i j}, \quad \alpha_{\lambda i j} \equiv 1 /\left(\alpha_{\mathrm{d} i j}^{4} \alpha_{\omega i j}^{2}\right),
$$

where $\lambda_{i j}$ is given by Eq. (12).

Another way to improve the ion-sphere rescaling for a pycnonuclear reaction in a BIM was proposed by Ichimaru et al. [51] who used the formal relation $\lambda_{i j} \sim$ $r_{\mathrm{B} i j} / d_{i j}$ and suggested that $\widetilde{\lambda}_{i j}=\lambda_{i j} / \alpha_{\mathrm{d} i j}$, which corresponds to $\alpha_{\lambda i j}=1 / \alpha_{\mathrm{d} i j}$. Therefore, they allowed $d_{i j}$ to be different from $d_{i j}^{(0)}$ but assumed that the oscillator frequency $\omega_{i j}$ adjusts to this new separation following the ion-sphere rescaling, so that $\alpha_{\omega i j}^{2}=1 / \alpha_{\mathrm{d} i j}^{3}$. In contrast, we allow the separations and oscillator frequencies to deviate independently, and our consideration is more general.

Following Ogata at al. [45] (see their Eq. (7)), we assume that the number of closest neighbors $j$ around the ion $i$ in Eq. (25) is $\left\langle\nu_{i j}\right\rangle_{\text {av }}=8 n_{j} / n$ (which is appropriate for a uniform mix). The factor ' 8 ' may be approximate but it affects only the pre-exponent of the reaction rate which is much less significant than the exponentially small probability of Coulomb tunneling. Substituting (27) and (28) into (25), we obtain

$$
\begin{aligned}
R_{i j}^{\mathrm{pyc}}= & D_{\mathrm{pyc}} \frac{4 n_{i} n_{j}}{1+\delta_{i j}} \frac{8\langle Z\rangle}{\left(Z_{i}^{1 / 3}+Z_{j}^{1 / 3}\right)^{3}} \frac{S\left(E_{i j}^{\mathrm{pk}}\right)}{\hbar} \\
& \times \frac{r_{\mathrm{B} i j}}{\widetilde{\lambda}_{i j}^{C_{\mathrm{pl}}}} \exp \left(-\frac{C_{\mathrm{exp}}}{\left(\widetilde{\lambda}_{i j}\right)^{1 / 2}}\right) .
\end{aligned}
$$

This equation has the same structure as Eq. (16) and can be written as

$$
R_{i j}^{\mathrm{pyc}}=\frac{n_{i} n_{j}}{1+\delta_{i j}} S\left(E_{i j}^{\mathrm{pk}}\right) \frac{r_{\mathrm{B} i j}}{\hbar} P_{\mathrm{pyc}} F_{\mathrm{pyc}}
$$

with

$$
P_{\mathrm{pyc}}=\frac{8\langle Z\rangle}{\left(Z_{i}^{1 / 3}+Z_{j}^{1 / 3}\right)^{3}} \frac{4 D_{\mathrm{pyc}}}{\widetilde{\lambda}_{i j}^{C_{\mathrm{pl}}}}, \quad F_{\mathrm{pyc}}=\left(-\frac{C_{\mathrm{exp}}}{\left(\widetilde{\lambda}_{i j}\right)^{1 / 2}}\right) .
$$

For numerical evaluations, we have

$$
\begin{aligned}
R_{i j}^{\mathrm{pyc}}= & 10^{46} C_{\mathrm{pyc}} \frac{8 \rho X_{N} x_{i} x_{j} A_{i} A_{j}\langle A\rangle Z_{i}^{2} Z_{j}^{2}}{\left(1+\delta_{i j}\right) A_{c}^{2}} S\left(E_{i j}^{\mathrm{pk}}\right) \\
& \times \widetilde{\lambda}_{i j}^{3-C_{\mathrm{pl}}} \exp \left(-\frac{C_{\mathrm{exp}}}{\left(\widetilde{\lambda}_{i j}\right)^{1 / 2}}\right) \mathrm{cm}^{-3} \mathrm{~s}^{-1}, \quad
\end{aligned}
$$

where $C_{\text {pyc }}=D_{\text {pyc }} /(8 \times 11.515)$; the density $\rho$ is expressed in $\mathrm{g} \mathrm{cm}^{-3}$ and the astrophysical factor $S\left(E_{i j}^{\mathrm{pk}}\right)$ is in $\mathrm{MeV}$ barn. The reaction energy is $E_{i j}^{\mathrm{pk}} \sim \hbar \omega_{i j}=$ $\alpha_{\omega i j} k_{\mathrm{B}} T_{i j}^{(p)}$. The main parameter regulating the reaction rate is $\tilde{\lambda}_{i j}$ in the exponent argument. For sufficiently low densities, $\widetilde{\lambda}_{i j}$ is very large, strongly suppressing the Coulomb tunneling. With growing $\rho$ the barrier becomes more transparent and the reaction rate increases.

For the OCP with $\alpha_{\lambda}=1$ Eqs. (30) (33) reduce to the well known equations for zero-temperature pycnonuclear burning in a crystalline lattice. The constants $C_{\mathrm{pyc}}, C_{\mathrm{pl}}$ and $C_{\exp }$, obtained using various techniques, have been analyzed in Ref. [1]. In Table II we present these parameters for three models. The first model is optimal (seems to be the most reliable). It is the static-lattice model of Salpeter and Van Horn [8] for the bcc crystal. The second and third models are phenomenological; they have been proposed in Ref. [11], basing on the results of Refs. [8, 9, 45, 47]. The second model gives the upper limit of the reaction rate and the third gives the lower limit (for both - bcc and fcc - crystals). We expect that the reaction rate in an amorphous OCP would lie within the same limits.

Returning to an MCP, we must additionally specify the scaling factor $\alpha_{\lambda i j}$ in Eq. (29). Some attempts have been made [45, 51] to determine proper inter-ion separations $d_{i j}$ (i.e., the values of $\alpha_{\mathrm{d} i j}$ ) at $T=0$ in BIM solids from positions of first correlation peaks in radial pair distribution functions of ions, $g_{i j}(r)$. These functions have been calculated by Monte Carlo sampling. Such studies require powerful computer resources and may be inconclusive at present. This is clearly seen from similar (and simpler) attempts of the same group 45, 46, 51, 52] to determine deviations of inter-ion separations from the ionsphere scaling in BIM liquids (using first-peak positions of $\left.g_{i j}(r)\right)$. The authors applied their results to study an effect of these deviations on thermonuclear burning in the strong screening regime. Our analysis of those results shows that no statistically significant deviations from the 
ion-sphere scaling have been found (and no associated effects on nuclear burning can actually be predicted). This conclusion is strengthened by the critical analysis of these works by Rosenfeld 49, 50]. Therefore, no reliable information has been obtained on the violation of the ionsphere scaling of inter-ion separations in the BIM liquids and solids (on the level of a few percent, which is most likely the real uncertainty in the determination of $g_{i j}(r)$ peak positions in the cited publications).

Equally, proper oscillator frequencies $\omega_{i j}$ (and parameters $\alpha_{\omega i j}$ ) in BIMs could be determined from molecular dynamics simulations but such simulations have not yet been performed.

In the absence of precise microscopic calculations of $d_{i j}$ and $\omega_{i j}$ we naturally assume that the optimal values are $\alpha_{\mathrm{d} i j}=\alpha_{\omega i j}=\alpha_{\lambda i j}=1$. In order to maximize the reaction rate we propose to increase $\alpha_{\lambda i j}$ (somewhat arbitrarily) by $5 \%$, and in order to minimize the rate we propose to reduce it by $5 \%$. These proposed values are also listed in Table III Notice that it is difficult to expect that the variations of $\alpha_{\mathrm{d} i j}$ and $\alpha_{\omega i j}$ in Eq. (28) are fully independent. An increase in the inter-ion separation $d_{i j}$ should cause a decrease in the oscillator frequency $\omega_{i j}$; these variations should be partially compensated in the factor $\alpha_{\lambda i j}=1 /\left(\alpha_{\mathrm{d} i j}^{4} \alpha_{\omega i j}^{2}\right)$.

\section{Regular MCP lattice}

Now we turn to the case a regular MCP lattice, which can be drastically different from a uniform mix. The central point is the availability of closest neighbors $i j$. If they are absent, the reaction (9) occurs via Coulomb tunneling of more distant $i j$ pairs and becomes strongly suppressed.

The closest-neighbor condition depends on the crystal type. For instance, consider a binary bcc crystal composed of ions $i$ and $j$. There are eight pairs of closest neighbors in a basic cubic cell (formed by one ion in the center of the cell and any other ion in a vertex). If all ions are of the same type (e.g., $\left.x_{i}=1\right)$, then all eight pairs participate in the same reaction $i i$ (the OCP case). In the BIM case $\left(x_{i}=x_{j}=\frac{1}{2}\right)$ we have an ion $i$ in the center of the cell and ions $j$ in vertices, and all eight pairs of closest neighbors participate only in the reaction $i j$. The reactions $i i$ and $j j$ will be strongly blocked because any pairs $i i$ and $j j$ are not closest neighbors. Then the ion $i$ in the center of the basic cell will be able to react with six ions $i$ in centers of adjacent cells. The equilibrium distance between these pairs is a factor of $2 / \sqrt{3} \approx 1.155$ larger, than between the closest neighbors, which will exponentially suppress the $i i$ reaction rate.

One can construct more complicated MCP lattice structures and formulate appropriate blocking conditions. The strongest blocking of pycnonuclear reactions is expected in a regular crystal with many components. In that case the probability to find specified closed neighbors $i j$ could be very selective.

\section{Other MCP structures}

If the matter consists of domains of separated phases, pycnonuclear burning occurs mainly within these domains. The rate of the reaction $i i$ in domains containing ions $i$ can be calculated from Eq. (33), assuming OCP $\left(x_{i}=1\right)$, and then diluted by a volume fraction occupied by the phase $i$. The burning of different ions $i$ and $j$ occurs on interfaces between corresponding domains. Roughly, in a BIM with spherical bubbles containing $N$ less abundant ions $(i$ or $j$ ) the OCP reaction rate $i i$ can be diluted with respect to the rate in a uniformly mixed state by a factor of $1 / N^{2 / 3}$ (because the reactions occur on interfaces).

Pycnonuclear burning in a lattice can be drastically affected by lattice impurities and imperfections (see, e.g., Ref. 8]). We expect that the effect of impurities or imperfections can be included in Eq. (33) if we treat them as members of the MCP. Because the burning is mainly regulated by the parameter $\widetilde{\lambda}_{i j}$, the rate should be extremely sensitive to variations of equilibrium distances and oscil-

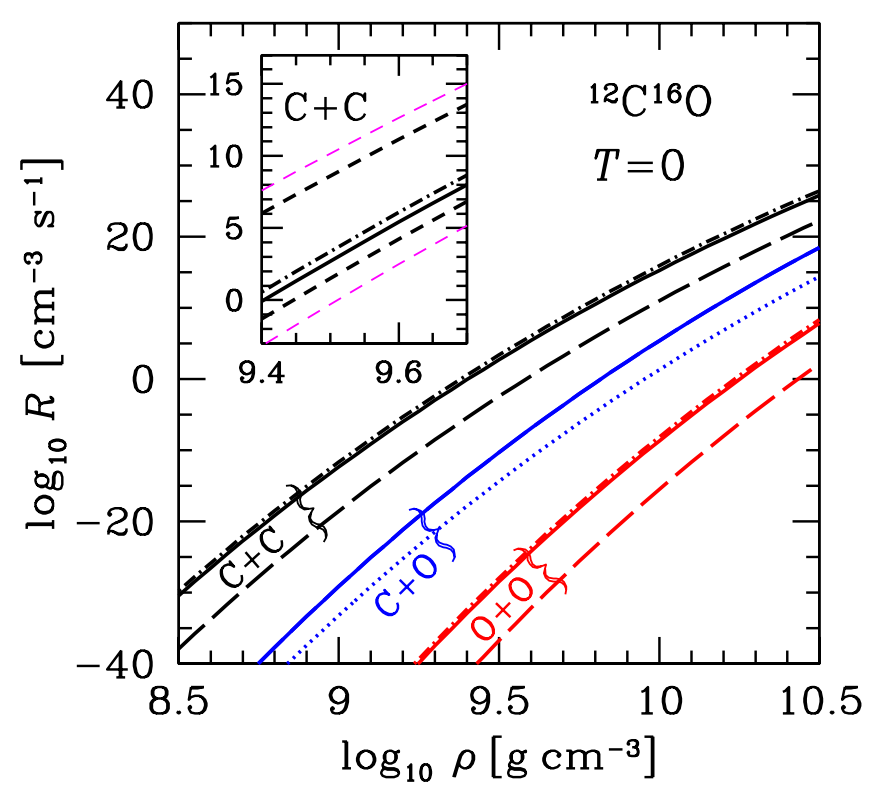

FIG. 3: (color online) Rates of pycnonuclear $\mathrm{C}+\mathrm{C}, \mathrm{C}+\mathrm{O}$ and $\mathrm{O}+\mathrm{O}$ reactions versus density for the optimal burning model. The dot-and-dashed lines show $\mathrm{C}+\mathrm{C}$ burning in a pure carbon crystal and $\mathrm{O}+\mathrm{O}$ burning in a pure oxygen crystal. Other lines are for the $\mathrm{CO}$ mixture $\left(x_{\mathrm{C}}=0.5\right)$; the solid lines refer to a uniform BIM; the long-dashed lines are for a regular $\mathrm{CO}$ bcc crystal; the dots are for phase-separated matter. The inset shows the same curves for the $\mathrm{C}+\mathrm{C}$ reaction on a larger scale; the thicker short-dashed lines give the maximum and minimum reaction rates for the uniform $\mathrm{CO}$ mixture in the ion-sphere model $\left(\alpha_{\lambda}=1\right)$; the thin short-dashed lines are the same but allowing for the variation of $\alpha_{\lambda}$ (see the text for details). 
lation frequencies for the reacting nuclei (see Eq. (29)). Small variations can induce exponentially huge jumps or drops of the Coulomb tunneling probability (for instance, in response to the decrease or increase of inter-ion separations). The impurities and imperfections may be rare but give the leading contribution into the reaction rate.

Moreover, different pairs $i j$ in an MCP may be exposed to different local conditions and have different separations and oscillator frequencies. One can incorporate these effects by introducing the averaging $\langle\ldots\rangle$ av over an ensemble of such pairs in Eq. (25).

For illustration, in Fig. 3 we show the density dependence of $\mathrm{C}+\mathrm{C}, \mathrm{C}+\mathrm{O}$ and $\mathrm{O}+\mathrm{O}$ pycnonuclear reactions in carbon-oxygen BIMs. The astrophysical factors are taken from Section [II In the main part of the figure we use the optimal reaction model from Table II The dot-and-dashed lines show the $\mathrm{C}+\mathrm{C}$ burning in a pure carbon matter $\left(x_{\mathrm{C}}=1\right)$ and $\mathrm{O}+\mathrm{O}$ burning in a pure oxygen matter $\left(x_{\mathrm{C}}=0\right)$ while other lines are for the $\mathrm{CO}$ mixture with $x_{\mathrm{C}}=\frac{1}{2}$. The solid lines present the reaction rates in the uniform $\mathrm{CO}$ mixture. For the $\mathrm{C}+\mathrm{C}$ and $\mathrm{O}+\mathrm{O}$ reactions these lines go slightly lower than the dash-and-dot lines because of the reduced amount of carbon and oxygen in a BIM (as compared to pure carbon or oxygen matter). The long-dashed lines give the reaction rates in the bcc $\mathrm{CO}$ regular crystal. The $\mathrm{C}+\mathrm{O}$ burning in this crystal has the same rate as in the $\mathrm{CO}$ uniform mix (the solid line), in our model. In order to illustrate the blocking effect (produced by the absence of closest $\mathrm{CC}$ or $\mathrm{OO}$ neighbors in the crystal) we have calculated the $\mathrm{C}+\mathrm{C}$ and $\mathrm{O}+\mathrm{O}$ reaction rates using Eq. (33) but increasing the distances between the reacting nuclei in Eq. (28) by a factor of $\alpha_{\mathrm{d} i i}=2 / \sqrt{3}$, where $i=\mathrm{C}$ or $\mathrm{O}$ (see above). Because the local oscillator frequencies for these $\mathrm{CC}$ and $\mathrm{OO}$ pairs are unknown, we have assumed the ion-sphere rescaling $\left(\alpha_{\omega i i}^{2}=1 / \alpha_{\mathrm{d} i i}^{3}\right)$. The blocking effect is strong, reducing the reaction rates by $5-7$ orders of magnitude. Nevertheless, even with this blocking, the $\mathrm{C}+\mathrm{C}$ reaction is much faster than the $\mathrm{C}+\mathrm{O}$ and $\mathrm{O}+\mathrm{O}$ ones because of much lower Coulomb barrier. Finally, we have also calculated the BIM reaction rates assuming phase separation of $\mathrm{C}$ and $\mathrm{O}$. We do not plot the respective $\mathrm{C}+\mathrm{C}$ and $\mathrm{O}+\mathrm{O}$ reaction rates because they are almost indistinguishable from the corresponding dotand-dashed lines (the rates are only twice lower than in the respective OCPs). However, the $\mathrm{C}+\mathrm{O}$ burning in this case can be strongly suppressed because it occurs only at interfaces between separated phases. For example, the dotted line shows such a $\mathrm{C}+\mathrm{O}$ burning rate assuming separation into domains which contain $10^{6}$ ions.

The inset in Fig. 3 displays the $\mathrm{C}+\mathrm{C}$ reaction rate on a larger scale. The solid and dot-and-dashed lines are the same as in the main part of the figure. Short dashed lines present the maximum and minimum reaction rates for the uniform $\mathrm{CO}$ mixture; they reflect uncertainties of existing theoretical models for pycnonuclear burning. These lines are calculated using the parameters from TableII Thicker short-dashed lines give the maximum and minimum reaction rates using the ion-sphere model (neglecting deviations from the ion-sphere rescaling; i.e., assuming $\alpha_{\lambda C C}=1$ ). Thinner short-dashed lines are the estimated maximum and minimum reaction rates taking into account possible deviations from the ion-sphere model. The current theoretical uncertainties of the reaction rate are really large.

\section{E. Thermally enhanced pycnonuclear regime}

This regime operates in the temperature range $0.5 T_{i j}^{(p)} / \ln \left(T_{i j}^{(l)} / T_{i j}^{(p)}\right) \lesssim T \lesssim 0.5 T_{i j}^{(p)}$ (see Ref. [8]). At these temperatures, the majority of nuclei occupy ground states in their potential wells but the main contribution into the reaction rate comes from a small amount of nuclei which occupy excited bound states. This regime is difficult for theoretical studies. We will follow the approach of Ref. 11], which is based on an analytical approximation of the WKB calculations performed by Salpeter and Van Horn [8]. We generalize this approach to an MCP in the same manner as in Section IIID by rescaling $\lambda$ and $T_{p}$ in accordance with Eqs. (12) and (10). In this case

$\frac{R_{i j}^{\mathrm{pyc}}(T)}{R_{i j}^{\mathrm{pyc}}(0)}-1=\frac{\Omega}{\widetilde{\lambda}_{i j}^{1 / 2}} \exp \left(-\Lambda \frac{\widetilde{T}_{i j}^{(p)}}{T}+\frac{\Omega_{1}}{\left(\widetilde{\lambda}_{i j}\right)^{1 / 2}} \mathrm{e}^{-\Lambda \widetilde{T}_{i j}^{(p)} / T}\right)$,

where $\widetilde{T}_{i j}^{(p)} \equiv \hbar \omega_{i j} / k_{\mathrm{B}}=\alpha_{\omega i j} T_{i j}^{(p)}$, while $\Omega, \Omega_{1}$, and $\Lambda$ are model-dependent dimensionless constants. We adopt $\alpha_{\omega i j}=0.95$ to maximize the reaction rate and $\alpha_{\omega i j}=$ 1.05 to minimize it (Table II). In analogy with Ref. 11 the characteristic energy of the reacting nuclei can be taken in the form

$$
E_{i j}^{\mathrm{pk}} \approx \hbar \omega_{i j}+\frac{Z_{i} Z_{j} e^{2}}{a_{i j}} \exp \left(-\Lambda \frac{\widetilde{T}_{i j}^{(p)}}{T}\right)
$$

The first term is the reaction energy in the zerotemperature pycnonuclear regime, while the second term describes an increase of $E_{i j}^{\mathrm{pk}}$ with growing temperature in the thermally enhanced pycnonuclear regime. Equation (35) is approximate (based on the results of Ref. 8 as explained in [1]]) but we expect that it correctly reflects the main features of the accurate expression (to be derived in future, more elaborated calculations). The analogous expression (26) for OCP in Ref. [11] contains two new free factors, $C_{1}$ and $C_{2}$, in the first and second terms, respectively. They were set $C_{1}=C_{2}=1$ in numerical calculations [11] because the theory [8] is insufficiently precise to determine them. We do not introduce these factors here to avoid additional unknowns, but they could be introduced in the future. An uncertainty in $E_{i j}^{\mathrm{pk}}$ should not greatly affect the reaction rates.

After Salpeter and Van Horn [8] the thermally enhanced pycnonuclear burning in an OCP was studied by Kitamura and Ichimaru [53] assuming that the reacting 
TABLE II: Coefficients in the interpolation expressions for a reaction rate for the optimal model of nuclear burning and for the models which maximize and minimize the rate. The parameters $C_{T}, \alpha_{\lambda i j}, \alpha_{\omega i j}$ are different for MCP and OCP (the values for OCP [1] are given in brackets). For an MCP, the models assume a uniformly mixed state (see the text for details).

\begin{tabular}{|c|c|c|c|c|c|c|c|}
\hline Model & $C_{\text {exp }}$ & $C_{\mathrm{pyc}}$ & $C_{\mathrm{pl}}$ & $C_{T}$ & $\alpha_{\lambda i j}$ & $\alpha_{\omega i j}$ & $\Lambda$ \\
\hline Optimal & 2.638 & 3.90 & 1.25 & $0.724(0.724)$ & $1 \quad(1)$ & $1 \quad(1)$ & 0.5 \\
\hline Maximum rate & 2.450 & 50 & 1.25 & $0.840(0.904)$ & $1.05(1)$ & $0.95(1)$ & 0.35 \\
\hline Minimum rate & 2.650 & 0.5 & 1.25 & $0.768(0.711)$ & $0.95(1)$ & $1.05(1)$ & 0.65 \\
\hline
\end{tabular}

nuclei move in an angle-averaged, radial static mean-field potential determined from Monte Carlo sampling of classical Coulomb systems. Although this approach is less justified than the WKB approximation of Ref. 8], the results are in a reasonable agreement (see Ref. 11 for details). Kitamura [4] generalized the results of Ref. 53] to the case of BIMs using the ion-sphere rescaling rule $\left(\alpha_{\lambda i j}=\alpha_{\omega i j}=1\right)$.

The thermally enhanced pycnonuclear burning is as sensitive to the microphysical structure of the MCP as the zero-temperature pycnonuclear burning (Section (IID). The above comments refer to a uniformly mixed MCP. In the case of a regular MCP lattice the reaction will be suppressed by the same blocking effects as discussed in Section IIID The case of phase separation has the same features as at $T=0$. Illustrative examples will be given in Section IV

\section{F. The intermediate thermo-pycnonuclear regime}

This regime is realized at temperatures $T_{i j}^{(p)} / 2 \lesssim T \lesssim$ $T_{i j}^{(p)}$ which separate the domains of classical and quantum motion of the reacting nuclei. The calculation of the reaction rate in this regime is most complicated. We will describe this rate by a phenomenological expression presented below.

The reaction is mainly determined by the nuclei which become slightly unbound and can move freely through the dense matter, fusing not only with the closest neighbors (pycnonuclear regime), but with other nuclei (thermonuclear regime). We expect that the transition from the pycnonuclear to the thermonuclear regime with the growth of temperature in a uniformly mixed MCP is sufficiently smooth. When the number of freely reacting nuclei becomes large, the dependence of the reaction rate on the details of the MCP microstructure should disappear.

\section{G. Single analytical approximation in all regimes}

Our phenomenological expression for the temperature and density dependent reaction rate, which combines all the five burning regimes and assumes a uniformly mixed $M C P$ at low temperatures, is a straitforward generaliza- tion of the expression for the OCP considered in Ref. [1],

$$
\begin{aligned}
& R_{i j}(\rho, T)=R_{i j}^{\mathrm{pyc}}(\rho)+\Delta R_{i j}(\rho, T), \\
& \Delta R_{i j}(\rho, T)=\frac{n_{i} n_{j}}{1+\delta_{i j}} \frac{S\left(E_{i j}^{\mathrm{pk}}\right)}{\hbar} r_{\mathrm{B} i j} P F \\
& F=\exp \left(-\widetilde{\tau}_{i j}+C_{\mathrm{sc}} \widetilde{\Gamma}_{i j} \varphi \mathrm{e}^{-\Lambda \widetilde{T}_{i j}^{(p)} / T}-\Lambda \frac{\widetilde{T}_{i j}^{(p)}}{T}\right), \\
& P=\frac{8 \pi^{1 / 3}}{\sqrt{3} 2^{1 / 3}}\left(\frac{E_{i j}^{\mathrm{a}}}{k_{\mathrm{B}} \widetilde{T}}\right)^{\gamma} .
\end{aligned}
$$

In this case, $\varphi=\sqrt{\Gamma_{i j}} /\left[\left(C_{i j}^{\mathrm{sc}} / \zeta_{i j}\right)^{4}+\Gamma_{i j}^{2}\right]^{1 / 4} ; R_{i j}^{\mathrm{pyc}}(\rho)$ is the density dependent pycnonuclear reaction rate at zero temperature discussed in Section IIID $\Delta R_{i j}(\rho, T)$ is the density and temperature dependent part including an exponential function $F$ and a pre-exponent $P$. The quantities $\widetilde{\tau}_{i j}$ and $\widetilde{\Gamma}_{i j}$ are similar to the familiar quantities $\tau_{i j}$ and $\Gamma_{i j}$, but contain a "renormalized" temperature $\widetilde{T}$,

$$
\begin{aligned}
& \widetilde{\tau}_{i j}=3\left(\frac{\pi}{2}\right)^{2 / 3}\left(\frac{E_{i j}^{\mathrm{a}}}{k_{\mathrm{B}} \widetilde{T}}\right)^{1 / 3}, \\
& \widetilde{\Gamma}_{i j}=\frac{Z_{i} Z_{j} e^{2}}{a_{i j} k_{\mathrm{B}} \widetilde{T}}, \quad \widetilde{T}=\sqrt{T^{2}+C_{T}^{2}\left(T_{i j}^{(p)}\right)^{2}},
\end{aligned}
$$

where $C_{T}$ is a dimensionless renormalization parameter specified below. Equations (36) and (37) are analogous to Eqs. (27) and (28) of Ref. [1]. The term $\Delta R_{i j}(\rho, T)$ and the renormalized temperature $\widetilde{T}$ are introduced to match the equations in thermonuclear and pycnonuclear regimes. The renormalized temperature reflects the fact that the thermal energy $k_{\mathrm{B}} T$ of plasma ions in the thermonuclear case is replaced by a temperature-independent zero-point energy in the pycnonuclear case.

For high temperatures $T \gg T_{i j}^{(p)}$ we have $\widetilde{\tau}_{i j} \rightarrow \tau_{i j}$, $\widetilde{\Gamma}_{i j} \rightarrow \Gamma_{i j}$, and $\widetilde{T} \rightarrow T$. In this case $\Delta R_{i j}(\rho, T) \rightarrow$ $R_{i j}^{\mathrm{th}}(\rho, T) \gg R_{i j}^{\mathrm{pyc}}(\rho)$, and Eq. (36) reproduces the thermonuclear reaction rate (Sections IIIB and IIC). At low temperatures $T \lesssim T_{i j}^{(p)}$ the quantities $\widetilde{\tau}_{i j}$, $\widetilde{\Gamma}_{i j}$ and $\widetilde{T}$ contain "the quantum temperature" $T_{i j}^{(p)}$, determined by zero-point ion vibrations, rather than the real temperature $T$. In the limit of $T \rightarrow 0$ we obtain $\widetilde{\Gamma}_{i j}=1 /\left[\left(\lambda_{i j}\right)^{1 / 2}(72 \pi)^{1 / 6} C_{T}\right]$ and $\widetilde{\tau}_{i j}=$ $3\left(\pi / \lambda_{i j}\right)^{1 / 2} /\left(2^{7 / 6} C_{T}^{1 / 3}\right)$. 
Following Ref. [11] we require that at $T \ll T_{i j}^{(p)}$ the factor $\exp \left(-\widetilde{\tau}_{i j}\right)$ in the exponential function $F$, Eq. (36), reduces to $\exp \left(-C_{\exp } /\left(\widetilde{\lambda}_{i j}\right)^{1 / 2}\right)$. This would allow us to obey Eq. (34) by satisfying the equality

$$
3 \sqrt{\pi} /\left(2^{7 / 6} C_{T}^{1 / 3}\right)=C_{\exp }\left(\alpha_{\lambda i j}\right)^{-1 / 2} .
$$

The double exponent factor in F, Eq. (36), will correspond to the double exponent factor in Eq. (34). Taking $C_{\exp }$ and $\alpha_{\lambda i j}$ from Table III we can determine $C_{T}$. These parameters are also listed in Table In the MCP they are different from those in the OCP because in the MCP we introduce an additional parameter $\alpha_{\lambda i j}$ (Section IIID). The values of $C_{T}$ for the OCP [11] are given in Table Iin brackets.

Finally, the quantity $\gamma$ in Eq. (36) and the reaction energy $E_{i j}^{\mathrm{pk}}$ in the astrophysical factor $S\left(E_{i j}^{\mathrm{pk}}\right)$ can be chosen in the same way as in Ref. 11],

$$
\begin{gathered}
\gamma=\left(T^{2} \gamma_{1}+\left(\widetilde{T}_{i j}^{(p)}\right)^{2} \gamma_{2}\right) /\left(T^{2}+\left(\widetilde{T}_{i j}^{(p)}\right)^{2}\right) \\
E_{i j}^{\mathrm{pk}}=\hbar \widetilde{\omega}_{i j}^{(p)}+\left(\frac{Z_{i} Z_{j} e^{2}}{a_{i j}}+\frac{k_{\mathrm{B}} T \tau_{i j}}{3}\right) \exp \left(-\frac{\Lambda \widetilde{T}_{i j}^{(p)}}{T}\right),
\end{gathered}
$$

where $\gamma_{1}=2 / 3$ and $\gamma_{2}=(2 / 3)\left(C_{\mathrm{pl}}+0.5\right)$.

Thus, we propose to use the analytic expression (36) for the reaction rate in a uniformly mixed MCP with the following parameters:

(1) The parameter $C_{\mathrm{sc}}$ of strongly screened thermonuclear burning is given by Eq. (21).

(2) The parameters $C_{\text {exp }}, C_{\text {pyc }}, C_{\mathrm{pl}}, \alpha_{\lambda i j}$ of zerotemperature pycnonuclear burning, and the parameters $\alpha_{\omega i j}, \Lambda$ and $C_{T}$ of thermally enhanced pycnonuclear burning are given in Table

In this way we obtain (Table III) three models for any given non-resonant nuclear fusion reaction (9) in a uniformly mixed MCP. One is the optimal model, the second gives the maximum reaction rate, and the third the minimum reaction rate. For the OCP, it is sufficient to set $\alpha_{\lambda i j}=\alpha_{\omega i j}=1$, which reduces the present results to the results of Ref. 11].

The uncertainties of the reaction rate become larger if a cold MCP forms a regular lattice or undergoes a phase separation or contains impurities and defects. All these cases can be approximately taken into account in the same way as discussed in Section IIID For instance, a reaction in a regular lattice can be strongly blocked by the absence of closest reacting neighbors (Sections IIID and IIIE also see Section [V].

Our formula for a uniformly mixed MCP gives a smooth behavior of the reaction rate as a function of temperature and density, without jumps at the solidification point (in analogy with an OCP, see Ref. [1]]). In the cases of other MCP microstructures such jumps may appear.
Our formula is flexible. Its parameters could be tuned when new microscopic calculations of reaction rates appear in the future. Moreover, the formula can be improved even if a new information on MCP properties (not on reaction rates directly) appear in the literature (for instance, on the deviations from the ion-sphere scaling at $T=0)$.

More complicated expressions for the reaction rates in the OCP and uniform BIMs were proposed by Kitamura 47]. His expressions are mainly based on the results of Refs. 45, 46, 48, 51, 53. (in the different regimes) which are not free of approximations (see Ref. 11] for details). His expressions for BIMs are obtained assuming the ion-sphere rescaling rule $\left(\alpha_{\lambda i j}=\alpha_{\omega i j}=1\right)$ and are, therefore, more restricted than our expression. Their derivation implies that they are valid for uniformly mixed BIMs. In particular, they do not take into account blocking effects in regular binary lattices.

In contrast to our formula, Kitamura took into account the effects of electron screening (finite polarizability of the electron gas). However, these effects are relatively weak; their strict inclusion in the pycnonuclear regime is complicated. We do not include them but, instead, take into account theoretical uncertainties of the reaction rates without electron screening. The results of Kitamura 47] for an OCP lie well within these uncertainties. His results for BIMs in the thermonuclear regime with strong screening and essentially different charges of reacting nuclei are less accurate than our results (Section $\amalg I C)$.

\section{NUCLEAR BURNING IN A CARBON-OXYGEN MIXTURE}

For illustration of our results, we analyze nuclear reactions in a dense ${ }^{12} \mathrm{C}^{16} \mathrm{O}$ mixture. Figure $\square$ shows the temperature dependence of the $\mathrm{C}+\mathrm{C}$ reaction rate at $\rho=5 \times 10^{9} \mathrm{~g} \mathrm{~cm}^{-3}$ (for the same reaction models as in Fig. 31). The dot-and-dashed line is the optimal model (Table III) for a pure carbon matter (from Ref. [11]). Other lines are for CO mixtures with equal numbers of $\mathrm{C}$ and $\mathrm{O}$ nuclei $\left(x_{\mathrm{C}}=\frac{1}{2}\right)$. The solid line is the optimal model for the uniform mixture; the thicker shortdashed lines show the maximum and minimum reaction rates in such a mixture in the ion-sphere approximation $\left(\alpha_{\lambda \mathrm{CC}}=\alpha_{\omega \mathrm{CC}}=1\right)$; the thinner short-dashed lines are the same but beyond the ion-sphere approximation (note that variations of $\alpha_{\omega \mathrm{CC}}$ appear to be much less important than variations of $\alpha_{\lambda \mathrm{CC}}$ ). The long-dashed line is for a CO regular lattice (in the same approximation which has been used in Fig. (3). A sharp jump of this curve is associated with the melting of the crystal (Fig. 2), which destroys the blocking of the $\mathrm{C}+\mathrm{C}$ burning and amplifies the reaction rate.

Figure 4 shows the reaction rates in all burning regimes (except for the classical thermonuclear burning which would require higher temperatures; see Fig. 21). The horizontal parts of the curves for $\log T[\mathrm{~K}] \lesssim 7.7$ refer 


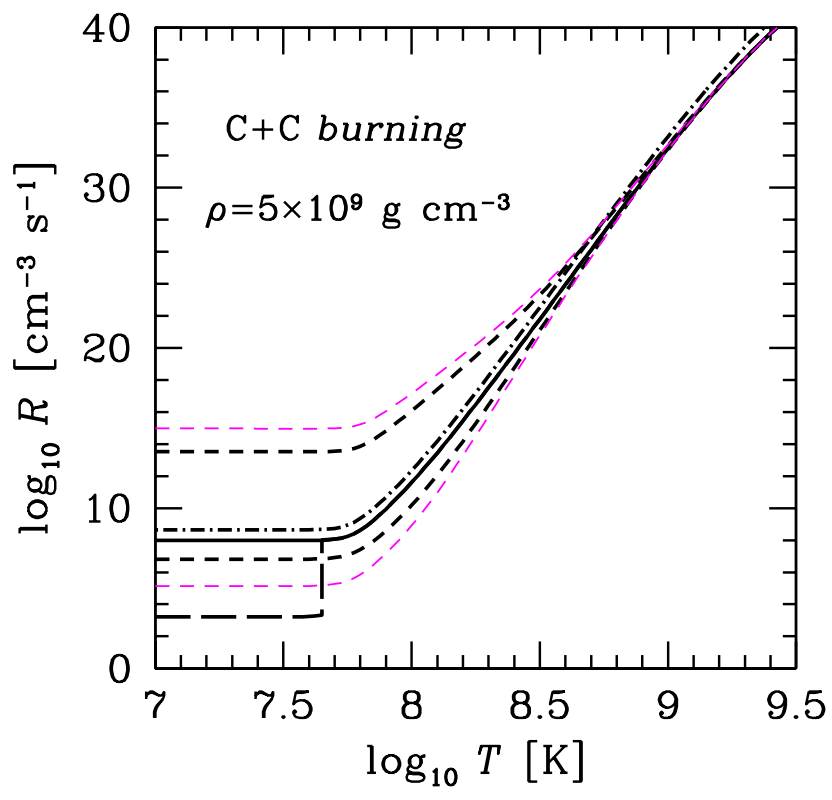

FIG. 4: (color online) The $\mathrm{C}+\mathrm{C}$ reaction rate versus temperature at $\rho=5 \times 10^{9} \mathrm{~g} \mathrm{~cm}^{-3}$. The dot-and-dashed line is the optimal model for a pure carbon matter; other lines are for a CO mixture with $x_{\mathrm{C}}=0.5$. The solid line is the optimal model for a uniform mixture; the long-dashed line is for the regular bcc CO crystal at low temperatures (the vertical part indicates the melting point). Thicker short-dashed lines give the maximum and minimum reaction rates for the uniform CO mixture in the ion-sphere model $\left(\alpha_{\lambda}=1\right)$; thinner short dashed-lines are the same but allowing for deviations from the ion-sphere model (see the text for details).

to the zero-temperature pycnonuclear burning; the respective reaction rates are independent of $T$ as discussed in Section IIID and displayed in Fig. 3. The temperature range $7.7 \lesssim \log T[\mathrm{~K}] \lesssim 8.3$ corresponds to the thermally enhanced pycnonuclear regime. The reaction rate starts to grow up with increasing $T$ (Section IIIE). The rate remains highly uncertain because of the same reasons as in the zero-temperature pycnonuclear regime. The next temperature range $8.3 \lesssim \log T[\mathrm{~K}] \lesssim 8.6$ corresponds to the intermediate thermo-pycnonuclear burning (Section IIIE). Theoretical uncertainties of the reaction rate become smaller. Finally, the temperature range $\log T[\mathrm{~K}] \gtrsim 8.6$ refers to the thermonuclear burning with strong plasma screening. The theoretical uncertainties become much smaller although the enhancement of the reaction rate by the plasma screening effects is huge; with increasing $T$ this enhancement weakens and the reaction rate matches the classical thermonuclear rate (see Fig. 6 of Ref. [1]]). The presence of oxygen slightly reduces the $\mathrm{C}+\mathrm{C}$ reaction rate (by reducing the amount of carbon nuclei at a given density).

Based on our expression for the reaction rates, in Fig. 2 we plot the $T-\rho$ domains (shaded strips), where the $\mathrm{C}+\mathrm{C}, \mathrm{C}+\mathrm{O}$ and $\mathrm{O}+\mathrm{O}$ reactions are most important. The

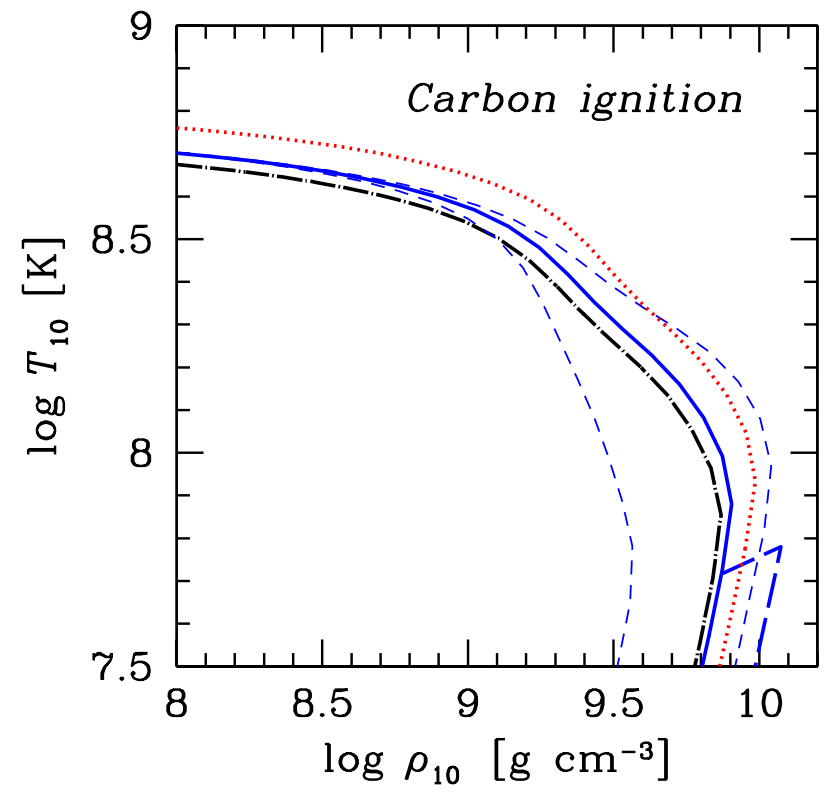

FIG. 5: (color online) Carbon ignition curves in ${ }^{12} \mathrm{C}{ }^{16} \mathrm{O}$ matter. The dot-and-dashed line is the optimal model for carbon burning in pure carbon matter. The solid and dotted lines are optimal models for uniform CO mixtures with $x_{\mathrm{C}}=0.5$ and 0.1 , respectively. Other lines are for CO BIMs with $x_{\mathrm{C}}=0.5$. The short-dashed lines give the highest and the lowest theoretical ignition curves for uniform mixtures. The long-dashed line is for the CO bcc crystal at low temperatures.

domains for the $\mathrm{C}+\mathrm{C}$ and $\mathrm{O}+\mathrm{O}$ reactions are presented for pure carbon or pure oxygen matter $\left(x_{\mathrm{C}}=1\right.$ and $x_{\mathrm{C}}=0$, respectively). For the $\mathrm{C}+\mathrm{O}$ reaction we have taken the $\mathrm{CO}$ mixture with $x_{\mathrm{C}}=\frac{1}{2}$. A domain for any reaction $i j$ is restricted by two lines, along which the characteristic burning time $\tau_{i}=n_{i} / R_{i j}$ of nuclei $i$ is constant (taken to be $10^{6}$ years for a lower line and 1 year for an upper line, for example). Above the upper line the reaction $i j$ is so fast, that the nuclei $i$ cannot survive for a long time. Below the lower line the reaction is so slow that the nuclei $i$ survive almost forever. Therefore, the strips represent the temperature and density domains of greatest relevance for the carbon and oxygen nucleosynthesis through the reactions under discussion. For determining these domains, we have taken the optimal model from Table III The domains do not change significantly under variations of fractional numbers of $\mathrm{C}$ and $\mathrm{O}$ within reasonable limits. For densities $\rho \lesssim 10^{9}$ $\mathrm{g} \mathrm{cm}{ }^{-3}$ the strips are almost horizontal; nuclear burning in them proceeds in the thermonuclear regime and the reaction rates depend mainly on the temperature. In contrast, the strips become almost vertical at low temperatures reflecting the pycnonuclear burning regime where the reaction rates depend mainly on the density.

The strips show a strong heterogeneity of the different reactions. It is evidently caused by different heights of 
Coulomb barriers. With increasing $\rho$ and/or $T$ in the $\mathrm{CO}$ matter, carbon will burn first in the $\mathrm{C}+\mathrm{C}$ reaction and could be burnt almost completely before reaching the $T-\rho$ domain, where the $\mathrm{C}+\mathrm{O}$ reaction can be efficient.

Finally, we have studied the carbon ignition curve, which is a necessary ingredient for modeling nuclear explosions of massive white dwarfs (supernova Ia events) and carbon explosions in accreting neutron stars (superbursts). The ignition curve is usually determined as the line in the $T-\rho$ plane (Fig. 5), where the nuclear energy generation rate equals the local neutrino energy losses. At higher $T$ and $\rho$ (above the curve) the nuclear energy generation exceeds the neutrino losses (which cool the matter) and carbon ignites. We have calculated such curves for $\mathrm{CO}$ mixtures. All reactions $(\mathrm{C}+\mathrm{C}, \mathrm{C}+\mathrm{O}$, and $\mathrm{O}+\mathrm{O})$ have been taken into account but the $\mathrm{C}+\mathrm{O}$ and $\mathrm{O}+\mathrm{O}$ reactions have appeared to be unimportant owing to the heterogeneity of nuclear burning. The presence of oxygen affects carbon ignition only through the $\mathrm{C}+\mathrm{C}$ reaction rate and the neutrino emission rate. The neutrino energy losses have been assumed to be produced by plasmon decay and by electron-nucleus bremsstrahlung. The neutrino emissivity owing to plasmon decay has been obtained from extended tables calculated by M. E. Gusakov (unpublished); they are in good agreement with the results of Itoh et al. [54]. The neutrino bremsstrahlung emissivity has been calculated using the formalism of Kaminker et al. [55], which takes into account electron band structure effects in crystalline matter. For a $\mathrm{CO}$ mixture, this neutrino emissivity has been determined using the linear mixture rule.

The dot-and-dashed line in Fig. [5] shows the carbon ignition curve, calculated using the optimal model of carbon burning in a pure carbon matter $\left(x_{\mathrm{C}}=1\right.$; from Ref. [1]). The solid and dotted lines are the same curves in CO mixtures with $x_{\mathrm{C}}=0.5$ and 0.1 , respectively (assuming the optimal reaction model and a uniform mixture at low temperatures). At $\rho \lesssim 10^{9} \mathrm{~g} \mathrm{~cm}^{-3}$ the curves depend weakly on the density because carbon burns in the thermonuclear regime. At $T \lesssim 10^{8} \mathrm{~K}$ the curves depend weakly on the temperature because carbon burns in the pycnonuclear regime. A strong bending of the curves in the density range from $\sim 10^{9} \mathrm{~g} \mathrm{~cm}^{-3}$ to $\sim 3 \times 10^{9} \mathrm{~g} \mathrm{~cm}^{-3}$ is associated with the transition from the thermonuclear burning to the pycnonuclear one. With decreasing the carbon fraction, the ignition curve shifts to higher $T$ and $\rho$, mainly because of the decrease of the $\mathrm{C}+\mathrm{C}$ reaction rate.

The short-dashed lines in Figure [5] show the uncertainty of the solid ignition curve $\left(x_{\mathrm{C}}=\frac{1}{2}\right.$, a uniform $\mathrm{CO}$ mixture) associated with the uncertainties of the reaction rates (assuming the maximum and minimum reaction rates from Table III). In the thermonuclear regime the uncertainties are small, while in the pycnonuclear regime they are substantial. The long-dashed line shows the ignition curve calculated under the assumption that a regular bcc $\mathrm{CO}$ lattice is formed in the $\mathrm{CO}$ mixture $\left(x_{\mathrm{C}}=\frac{1}{2}\right)$ after the crystallization. The blocking of the
$\mathrm{C}+\mathrm{C}$ reaction rate by oxygen ions in the bcc lattice shifts the ignition curve to higher $\rho$. The sudden break of this line is associated with the crystallization (analogous to the break of the long-dashed line in Fig. 4).

The carbon ignition curve obtained by equating the nuclear energy generation and the neutrino losses becomes unreliable for $T \lesssim 10^{8} \mathrm{~K}$ (e.g., Ref. [11]). The main reason is that this curve falls in the $T-\rho$ domain, where the characteristic carbon burning time is unrealistically large (exceeds the Universe age). In addition, the neutrino emission becomes very slow, inefficient for carrying away the nuclear energy; thermal conduction can be much more efficient. As a result, the carbon ignition condition becomes nonlocal, complicated, and dependent on a specific model (a neutron star or a white dwarf, etc.).

\section{CONCLUSIONS}

We have studied the problem of Coulomb barrier penetration for non-resonant nuclear fusion reactions in a dense MCP of atomic nuclei. We have considered all five nuclear burning regimes (Sections IIIB ПIIF) and analyzed calculations of nuclear reaction rates in an MCP for these regimes, available in the literature. We have proposed (Section IIG) a unified phenomenological expression for the reaction rate valid for all regimes. It generalizes an analogous expression proposed recently [1] for an OCP. The expression contains several parameters which can be varied to account for current theoretical uncertainties of the reaction rates.

Our main conclusions are:

1. The reaction rates in the thermonuclear regimes (with weak and strong plasma screening) can be calculated sufficiently accurately. In the regime of strong screening and for reacting nuclei with nonequal charges, our expression is more accurate than those proposed in the literature (Section IIIC).

2. The reaction rates in other regimes (zerotemperature and thermally enhanced pycnonuclear regimes; intermediate thermo-pycno nuclear regime) are much less certain. They are very sensitive to currently unknown microphysical correlation properties in an MCP (a uniform mix, a regular crystalline lattice, a phase separated matter, a matter with impurities and defects); they are much richer in physics than in the OCP case.

3. At low temperatures, we have mainly considered reactions in a uniform mix. Other MCP microstructures can strongly decrease or increase the reaction rates. For instance, the reactions in a regular MCP lattice can be strongly suppressed by the absence of nearby reacting nuclei.

4. Our phenomenological expression can be improved (Section IIG) after new calculations of the reac- 
tion rates or main properties of the MCP are performed. It would be important to know the actual microstructure of the MCP at low temperatures (first of all, the availability of closest neighbors, local separations and oscillation frequencies of neighboring nuclei, particularly in the presence of impurities and lattice defects).

5. Although our main formula in Section IIIG assumes a uniform mix at low $T$, the presented results are sufficient to understand qualitatively the reaction rates for other cases (following prescriptions of Section [IID.

For illustration, we have considered (Section IV) $\mathrm{C}+\mathrm{C}$, $\mathrm{C}+\mathrm{O}$, and $\mathrm{O}+\mathrm{O}$ nuclear reactions in a dense carbonoxygen mixture, that is important for the structure and evolution of massive white dwarfs (supernova Ia explosions) and accreting neutron stars (as sources of superbursts). For this purpose we have calculated and parameterized the appropriate astrophysical factors (Section II). The main results of our analysis are as follows:

1. The ranges of densities and temperatures, where $\mathrm{C}+\mathrm{C}, \mathrm{C}+\mathrm{O}$, and $\mathrm{O}+\mathrm{O}$ reactions are most important, look like narrow regions in the temperaturedensity diagram (Fig. 2); the regions do not strongly overlap which means strong heterogeneity of these reactions.

2. With increasing density and/or temperature, carbon starts burning first in the $\mathrm{C}+\mathrm{C}$ reaction (because carbon nuclei have lower Coulomb barrier); this reaction is most important for the nuclear evolution of $\mathrm{CO}$ mixtures.
3. Carbon burning in the $\mathrm{C}+\mathrm{C}$ reaction is affected by the presence of oxygen. The effect is simple in the thermonuclear regimes but more complicated in other regimes (at low temperatures).

4. Carbon ignition in a CO mixture occurs (Fig. 5) in thermonuclear regimes as long as $\rho \lesssim 10^{9} \mathrm{~g} \mathrm{~cm}^{-3}$ (and $T \gtrsim 3 \times 10^{8} \mathrm{~K}$ ). It can be calculated quite accurately. With decreasing the fraction of carbon, the ignition curve shifts to higher $\rho$ and $T$.

5. At $\rho \gtrsim 10^{9} \mathrm{~g} \mathrm{~cm}^{-3}$ and $T \lesssim 3 \times 10^{8} \mathrm{~K}$ the ignition condition becomes uncertain (Section IV). The formation of a regular CO lattice after the crystallization can block the $\mathrm{C}+\mathrm{C}$ reaction and shift the carbon ignition to higher densities.

Our consideration in this paper was general. More quantitative nuclear network simulations involving thermonuclear and pycnonuclear burning in dense stellar matter are currently in progress.

\section{Acknowledgments}

We are grateful to H. E. DeWitt for comments and suggestions and to M. E. Gusakov for providing the tables of neutrino emissivities due to plasmon decay. This work was partially supported by The Joint Institute for Nuclear Astrophysics (JINA) NSF PHY 0216783, by the Russian Foundation for Basic Research (grants 05-0216245, 05-02-22003) and by the Federal Agency for Science and Innovations (grant NSh 9879.2006.2).
[1] M. F. El Eid, B. S. Meyer, and L.-S. The, Astrophys. J. 611452 (2004).

[2] R. C. Pardo, R. G. Couch, and W. D. Arnett, Astrophys. J. 191, 711 (1974).

[3] J. W. Truran and W. D. Arnett, Astrophys. J. 160, 181 (1970).

[4] J. C. Niemeyer and S. E. Woosley, Astrophys. J. 475740 (1997).

[5] T. Strohmeyer and L. Bildsten, in Compact Stellar X-Ray Sources, eds. W. H. G. Lewin, M. Van der Klis (Cambridge University Press, Cambridge) astro-ph/0301544

[6] A. Cumming, J. Macbeth, J. J. M. in 't Zand, and D. Page, Astrophys. J. 646429 (2006).

[7] D. Page and A. Cumming, Astrophys. J. 635, L157 (2005)

[8] E. E. Salpeter and H. M. Van Horn, Astrophys. J. 155, 183 (1969).

[9] S. Schramm and S. E. Koonin, Astrophys. J. 365, 296 (1990); erratum: 377, 343 (1991).

[10] P. Haensel and J. L. Zdunik, Astron. Astrophys. 229, 117 (1990); P. Haensel and J. L. Zdunik, Astron. Astrophys. 404, L33 (2003).
[11] L. R. Gasques, A. V. Afanasjev, E. F. Aguilera, M. Beard, L. C. Chamon, P. Ring, M. Wiescher, and D. G. Yakovlev, Phys. Rev. C72, 025806 (2005).

[12] W. A. Fowler, G. R. Caughlan, and B. A. Zimmerman, Ann. Rev. Astr. Ap. 13, 69 (1975)

[13] D. D. Clayton, Principles of Stellar Evolution and Nucleosynthesis (University of Chicago Press, Chicago, 1983).

[14] E. E. Salpeter, Australian J. Phys. 7, 373 (1954).

[15] L. R. Gasques, L. C. Chamon, D. Pereira, M. A. G. Alvarez, E. S. Rossi Jr., C. P. Silva and B. V. Carlson, Phys. Rev. C69, 034603 (2004).

[16] M. A. Cândido Ribeiro, L. C. Chamon, D. Pereira, M. S. Hussein, and D. Galetti, Phys. Rev. Lett. 78, 3270 (1997).

[17] L. C. Chamon, D. Pereira, M. S. Hussein, M. A. Cândido Ribeiro, and D. Galetti, Phys. Rev. Lett. 79, 5218 (1997).

[18] L. C. Chamon, D. Pereira, and M. S. Hussein, Phys. Rev. C58, 576 (1998).

[19] L. C. Chamon, B. V. Carlson, L. R. Gasques, D. Pereira, C. De Conti, M. A. G. Alvarez, M. S. Hussein, M. A. Cândido Ribeiro, E. S. Rossi Jr., and C. P. Silva, Phys. Rev. C66, 014610 (2002). 
[20] M. A. G. Alvarez et al., Phys. Rev. C65, 014602 (2002).

[21] L. R. Gasques, L. C. Chamon, C. P. Silva, D. Pereira, M. A. G. Alvarez, E. S. Rossi Jr., V. P. Likhachev, B. V. Carlson, and C. De Conti, Phys. Rev. C65, 044314 (2002).

[22] E. S. Rossi Jr., D. Pereira, L. C. Chamon, C. P. Silva, M. A. G. Alvarez, L. R. Gasques, J. Lubian, B. V. Carlson, and C. De Conti, Nucl. Phys. A707, 325 (2002).

[23] C. A. Barnes, Essays in Nuclear Astrophysics eds. C. A. Barnes, D. D. Clayton, D. N. Schramm (Cambridge University Press, Cambridge, 1982), p. 193.

[24] J. R. Patterson, H. Winkler and C. S. Zaidins, Astrophys. J. 157, 367 (1969).

[25] M. G. Mazarakis and W. E. Stephens, Phys. Rev. C7, 1280 (1973).

[26] M. D. High and B. Cujec, Nucl. Phys. A282, 181 (1977).

[27] P. Rosales et al., Rev. Mex. Fís. 49, 88 (2003).

[28] K. U. Kettner, H. Lorenz-Wirzba and C. Rolfs, Z. Phys. A298, 65 (1980).

[29] H. W. Becker et al., Z. Phys. A303, 305 (1981).

[30] P. R. Christensen, Z.E. Switkowskiw, and R. A. Dayras, Nucl. Phys. A280, 189 (1977).

[31] B. Cujec and C. A. Barnes, Nucl. Phys. A266, 461 (1976).

[32] G. Hulke, C. Rolfs, and H.-P. Trautvetter, Z. Physik A297, 161 (1980).

[33] A. Kuronen, J. Keinonen, and P. Tikkanen, Phys. Rev. C35, 591 (1987).

[34] S.-C. Wu and C. A. Barnes, Nucl. Phys. A422, 373 (1984).

[35] D. G. Kovar et al., Phys. Rev. C20, 1305 (1979).

[36] J. Thomas, Y. T. Chen, S. Hinds, D. Meredith, and M. Olson, Phys. Rev. C33, 1679 (1986).

[37] H. E. DeWitt, W. L. Slattery, and J. Yang, in Strongly Coupled Plasma Physics, eds. H. M. Van Horn and
S. Ichimaru (University of Rochester Press, Rochester, 1993) p. 425.

[38] H. DeWitt and W. Slattery, Contrib. Plasma Phys. 43, 279 (2003)

[39] H. E. DeWitt, W. Slattery, D. Baiko and D. Yakovlev, Contrib. Plasma Phys. 41, 251 (2001).

[40] S. Ichimaru, Rev. Mod. Phys. 54, 1017 (1982).

[41] H. E. DeWitt, H. C. Graboske and M. S. Cooper, Astrophys. J. 181, 439 (1973).

[42] B. Jancovici, J. Stat. Phys. 17, 357 (1977).

[43] R. Mochkovitch and K. Nomoto, Astron. Astrophys. 154, 115 (1986); erratum: 157, 403 (1986).

[44] H. DeWitt and W. Slattery, Contrib. Plasma Phys. 39, 97 (1999).

[45] S. Ogata, H. Iyetomi, and S. Ichimaru, Astrophys. J. 372, 259 (1991).

[46] S. Ogata, S. Ichimaru, and H. M. Van Horn, Astrophys. J. 417, 265 (1993).

[47] H. Kitamura, Astrophys. J. 539, 888 (2000).

[48] S. Ogata, Astrophys. J. 481, 883 (1997).

[49] Y. Rosenfeld, Phys. Rev. E53, 2000 (1996).

[50] Y. Rosenfeld, Phys. Rev. E54, 2827 (1996).

[51] S. Ichimaru, S. Ogata, and H. M. Van Horn, Astrophys. J. 401, L35 (1992).

[52] S. Ogata, H. Iyetomi, S. Ichimaru, and H. M. Van Horn, Phys. Rev. E48, 1344 (1993).

[53] H. Kitamura and S. Ichimaru, Astrophys. J. 438, 300 (1995).

[54] N. Itoh, H. Mutoh, A. Hikita, and Y. Kohyama, Astrophys. J. 395, 622 (1992); erratum: 404, 418 (1993).

[55] A. D. Kaminker, C. J. Pethick, A. Y. Potekhin, V. Thorsson, and D. G. Yakovlev, Astron. Astrophys. 343, 1009 (1999). 